ESAIM: M2AN

Vol. 40, No 6, 2006, pp. 961-990

DOI: $10.1051 / \mathrm{m} 2 \mathrm{an}: 2007004$
ESAIM: Mathematical Modelling and Numerical Analysis

www.edpsciences.org/m2an

\title{
THEORETICAL AND NUMERICAL STUDY OF A QUASI-LINEAR ZAKHAROV SYSTEM DESCRIBING LANDAU DAMPING
}

\author{
R. Belaouar ${ }^{1,2}$, T. Colin $^{2,3}$, G. Gallice ${ }^{1}$ and C. Galusinski ${ }^{2,3}$
}

\begin{abstract}
In this paper, we study a Zakharov system coupled to an electron diffusion equation in order to describe laser-plasma interactions. Starting from the Vlasov-Maxwell system, we derive a nonlinear Schrödinger like system which takes into account the energy exchanged between the plasma waves and the electrons via Landau damping. Two existence theorems are established in a subsonic regime. Using a time-splitting, spectral discretizations for the Zakharov system and a finite difference scheme for the electron diffusion equation, we perform numerical simulations and show how Landau damping works quantitatively.
\end{abstract}

Mathematics Subject Classification. 35Q60, 65T50, 65M06.

Received: May 24, 2006. Revised: July 31, 2006.

\section{INTRODUCTION AND PHYSICAL SITUATION}

The interaction of an intense laser pulse with a plasma is a complex physical phenomenon where numerical simulation plays a key role in its understanding. One of the main goal is to simulate nuclear fusion by inertial confinement in a laboratory. We therefore need some accurate and reliable numerical models of laser-plasma interactions. Vlasov or particle-in-cell (PIC) simulations have been used for a more complete description of the problem. However, these kinetic simulations have difficulties in studying weak instabilities and long time behaviors because they need to resolve very small spatial and temporal scales. For the same reasons, it is not possible to use Euler-Maxwell equations. At the beginning of the 70's, Zakharov and its collaborators introduced the so-called Zakharov's equations in order to describe the non-linear interactions between the high-frequency electronic plasma waves and the low-frequency ion-acoustic waves. Basically, the slowly varying envelope of the electric field $E=\nabla \psi$ is coupled to the low-frequency variation of the density of the ions $\delta n$ by the following equations written in a dimensionless form [21]:

$$
\left\{\begin{array}{l}
i \partial_{t} \nabla \psi+\Delta(\nabla \psi)=\nabla \Delta^{-1} \operatorname{div}(\delta n \nabla \psi), \\
\partial_{t}^{2} \delta n-\Delta \delta n=\Delta\left(|\nabla \psi|^{2}\right) .
\end{array}\right.
$$

Keywords and phrases. Landau damping, Zakharov system.

1 SIS, CEA CESTA, BP 2, 33114 Le Barp, France.

2 Mathématiques Appliquées de Bordeaux, UMR CNRS 5466 et CEA LRC M03, Université Bordeaux 1, 351 cours de la Libération, 33405 Talence, France. Thierry.Colin@math.u-bordeaux1.fr

3 INRIA Futurs, project MC2. 
Of course, variations of this systems exist (see [19] for example). For laser propagation, one uses the paraxial approximation and the Zakharov system reads

$$
\left\{\begin{array}{l}
i\left(\partial_{t}+\partial_{y}\right) E+\Delta_{\perp} E=n E \\
\left(\partial_{t}^{2}-\Delta_{\perp}\right) n=\Delta_{\perp}\left(|E|^{2}\right)
\end{array}\right.
$$

where $\Delta_{\perp}=\partial_{x}^{2}+\partial_{z}^{2}$. (See [14] for a systematic use of this kind of models for numerical simulation). Concerning the system $(1.2)$, Linares et al. (see $[10,11]$ ) have shown that the Cauchy problem is well-posed in $H^{s}\left(\mathbb{R}^{n}\right)$ but Colin and Metivier (see [4]) have shown that it is ill-posed in $H^{s}\left(\mathbb{T}^{n}\right)$, where $\mathbb{T}^{n}$ denotes the $n$-dimensional torus.

Recently, Colin and Colin [3], starting from [15], derived a complete set of quasi-linear Zakharov equations describing the interactions between the laser fields, the stimulated Raman and Brillouin processes, the electronic plasma waves and the low-frequency variations of density of the ions. The system involves four Schrödinger equations coupled by quasi-linear terms and a wave equation. It reads:

$$
\begin{aligned}
& i\left(\partial_{t}+\frac{k_{0} c^{2}}{\omega_{0}} \partial_{y}\right) A_{0}+\frac{c^{2}}{2 \omega_{0}} \Delta A_{0}-\frac{k_{0}^{2} c^{4}}{2 \omega_{0}^{3}} \partial_{y}^{2} A_{0}=\frac{\omega_{p e}^{2}}{2 \omega_{0}} \delta n\left(A_{0}+\mathrm{e}^{-2 i k_{0} y} A_{B}\right) \\
& -\frac{e}{2 m_{e} \omega_{0}}\left(\nabla \cdot E_{0}\right) A_{R} \mathrm{e}^{-i\left(k_{1} y-\omega_{1} t\right)}, \\
& i\left(\partial_{t}-\frac{k_{0} c^{2}}{\omega_{0}} \partial_{y}\right) A_{B}+\frac{c^{2}}{2 \omega_{0}} \Delta A_{B}-\frac{k_{0}^{2} c^{4}}{2 \omega_{0}^{3}} \partial_{y}^{2} A_{B}=\frac{\omega_{p e}^{2}}{2 \omega_{0}} \delta n\left(A_{0}+\mathrm{e}^{-2 i k_{0} y} A_{B}\right) \\
& -\frac{e}{2 m_{e} \omega_{0}}\left(\nabla \cdot E_{0}\right) A_{R} \mathrm{e}^{i\left(\left(2 k_{0}-k_{1}\right) y-\omega_{1} t\right)}, \\
& i\left(\partial_{t}+\frac{k_{R} c^{2}}{\omega_{R}} \partial_{y}\right) A_{R}+\frac{c^{2}}{2 \omega_{R}} \Delta A_{R}-\frac{k_{R}^{2} c^{4}}{2 \omega_{R}^{3}} \partial_{y}^{2} A_{R}=\frac{\omega_{p e}^{2}}{2 \omega_{R}} \delta n A_{R} \\
& -\frac{e}{2 m_{e} \omega_{R}}\left(\nabla \cdot E_{0}^{*}\right)\left(A_{0}+\mathrm{e}^{-2 i k_{0} y} A_{B}\right) \mathrm{e}^{i\left(k_{1} y-\omega_{1} t\right)}, \\
& i \partial_{t} E_{0}+\frac{v_{t h}^{2}}{2 \omega_{p e}} \Delta E_{0}=\frac{\omega_{p e}}{2} \nabla \Delta^{-1} \operatorname{div}\left(\delta n E_{0}\right)+\frac{e \omega_{p e}}{2 c^{2} m_{e}} \nabla\left(A_{R}^{*}\left(A_{0}+\mathrm{e}^{-2 i k_{0} y} A_{B}\right) \mathrm{e}^{i\left(k_{1} y-\omega_{1} t\right)}\right) \\
& \left(\partial_{t}^{2}-c_{s}^{2} \Delta\right) \delta n=\frac{1}{4 \pi n_{0} m_{i}} \Delta\left(\left|E_{0}\right|^{2}+\frac{\omega_{p e}^{2}}{c^{2}}\left(\left|A_{0}+\mathrm{e}^{-2 i k_{0} y} A_{B}\right|^{2}+\left|A_{R}\right|^{2}\right)\right) \text {. }
\end{aligned}
$$

Here $A_{0}$ is the incident laser field, $A_{B}$ is the Brillouin component, $A_{R}$ is the Raman field, $E_{0}$ the electronicplasma field and $\delta n$ the low-frequency variation of the density of the ions. See [3] for the precise definition of all the constants involved in (1.3)-(1.7). Recall that the Raman and Brillouin processes are instabilities that occur during the propagation of a laser in a nonlinear medium. These instabilities are responsible for the creation of new waves (the Raman and Brillouin components) and correspond to a 3-waves interaction.

However these various fluid models do not take into account the kinetic effects such that Landau damping. The Landau damping corresponds to an exchange of energy between plasma waves and electrons that can reach high temperatures. The aim of this paper is to give a quantitative description of this phenomena. This process is especially important in the context of fusion by inertial confinement by lasers because electrons are accelerated to high energy and this induces a preheat of the fusion fuel and reduces the target gain. In order to obtain a system describing this wave-particle process we will derive a new set of equations starting from the VlasovMaxwell system. This will be done in Section 2. The system involves the Zakharov equations coupled with a 
quasi-linear diffusion equations for the electron distribution function. In one space dimension, the dimensionless system reads:

$$
\begin{aligned}
& i\left(\partial_{t} E+\nu \star E\right)+\partial_{x}^{2} E=\delta n E+E_{p}(x) \mathrm{e}^{i\left(k_{1} x-\omega_{1} t\right)}, \\
& \partial_{t}^{2} \delta n-\mu \partial_{x}^{2} \delta n=\mu \partial_{x}^{2}\left(|E|^{2}\right), \\
& \widehat{\nu}(t, \xi)=-\frac{\pi}{2 \xi|\xi|} \partial_{v} F_{e}\left(t, v=\frac{1}{\xi}\right), \xi \in \Omega_{\xi},
\end{aligned}
$$

where

$$
\partial_{t} F_{e}=\partial_{v}\left(D(t, v) \partial_{v} F_{e}\right), \quad D(t, v)=\frac{1}{4|v|}\left|\widehat{E}\left(t, \xi=\frac{1}{v}\right)\right|^{2}, v \in \Omega_{v}
$$

Here $E$ is the slowly varying amplitude of the high-frequency electronic plasma waves, $\delta n$ the low-frequency variation of the density of the ions, $F_{e}$ the spatially average electron distribution function and $\widehat{\nu}$ the spatial Fourier transform of $\nu$ corresponding to the Landau damping rate. This kind of model is valid for bounded velocity that are also bounded away from zero. $\Omega_{v}$ is the velocity domain on which equation (1.11) has to be satisfied and $\Omega_{\xi}=\left\{\xi \in \mathbb{R}\right.$ s.t. $\left.\exists v \in \Omega_{v}, v=\frac{1}{\xi}\right\}$. The domain $\Omega_{\xi}$ will therefore be taken under the form, $\Omega_{\xi}=[-A,-a] \cup[a, A]$ with $0<a<A$ (see Sect. 2).

Note the term $\widehat{\nu}$ is only defined on $\Omega_{\xi}$ by (1.10) and is extended by 0 outside the domain $\Omega_{\xi}$.

The term $E_{p}(x) \mathrm{e}^{i\left(k_{1} x-\omega_{1} t\right)}$ is the pump wave. In this work, it is a given function. $\left(k_{1}, \omega_{1}\right)$ satisfies the dispersion relation of the Schödinger equation: $\omega_{1}=\frac{3}{2} k_{1}^{2}$. The energy is brought to the system through this term. In fact, in a more complete model, this term will be given by the Raman interaction given by system (1.3)-(1.7) and is equal to $\nabla\left(A_{R}^{*}\left(A_{0}+\mathrm{e}^{-2 i k_{0} y} A_{B}\right) \mathrm{e}^{i\left(k_{1} y-\omega_{1} t\right)}\right)$. We postponed the study of this completed system to a future work.

\subsection{Statements of the results}

The local in time Cauchy problem for the usual Zakharov equations (1.1) is now well understood in the context of regular solutions (see $[1,12,17,18]$ for local models, see [2] for the non-local case (1.1)). For weak solutions, one can see [6]. For finite-time blow-up see [7,8]. For system (1.3)-(1.6), local existence in time for strong solutions is shown in [3].

Unfortunately, we are not able at this point to give an existence result for (1.8)-(1.11). We will restrict ourself to the case $\mu=+\infty$ corresponding to a "subsonic regime". In this case, introducing $H_{e}(t, \xi)=F_{e}\left(t, \frac{1}{\xi}\right)$ and denoting by $\Omega=\Omega_{\xi}$ the frequency domain, system (1.8)-(1.11) becomes

$$
\begin{aligned}
& i\left(\partial_{t} E+\nu * E\right)+\partial_{x}^{2} E=|E|^{2} E+f, \\
& \partial_{t} H_{e}-\xi^{2} \partial_{\xi}\left(|\xi|^{3}|\hat{E}|^{2} \partial_{\xi} H_{e}\right)=0, \quad \forall \xi \in \Omega, \\
& \hat{\nu}(t, \xi)=\operatorname{sgn}(\xi) \partial_{\xi} H_{e}(t, \xi) 1_{\Omega}, \\
& H_{e}(0, .)=H_{e 0}(.), E(0, .)=E_{0}(.) .
\end{aligned}
$$

The second equation of system (1.12) has to be endowed with boundary conditions. Since $\widehat{\nu}$ is extended by zero outside of $\Omega$, and since $\widehat{\nu}(t, \xi)=\operatorname{sgn}(\xi) \partial_{\xi} H_{e}(t, \xi)$ for $\xi \in \Omega$, it is natural to impose $\partial_{\xi} H_{e \mid \partial \Omega}=0$.

In order to construct local in time solutions for (1.12), the main problem is to deal with a nonlinear coupling between the electric field, which is a function of the space position, and the electronic distribution, which is function of the frequency. Due to this spatio-frequential coupling as well as the nonlinear terms, we need to simultaneously consider the problem in space and frequency variables for the electric field. 
We obtain two kinds of result. The first one concerns the local existence and uniqueness of solutions to (1.12). Moreover, one shows that if $\widehat{\nu}(0, \xi) \geq 0$ then for all $t, \widehat{\nu}(t, \xi) \geq 0$. The term $\nu * E$ is therefore a damping term. The second result is a global existence result but only in the case where the nonlinear term $|E|^{2} E$ and the source term $f$ in the right-hand-side of the first equation of (1.12) are replaced by 0 . Note that even in this case, the system is far away of being linear!

Let us denote by $H_{n}^{2}(\Omega)=\left\{g \in H^{2}(\Omega)\right.$ s.t. $\frac{\partial g}{\partial x}=0$ on $\left.\partial \Omega\right\}$.

Theorem 1.1 (local strong solutions). Let $E_{0}$ and $H_{e 0}$ such that $E_{0} \in H^{1}(\mathbb{R}), \hat{E}_{0} \in H^{2}(\Omega) \cap H^{1}(\mathbb{R})$ and $H_{e 0} \in H_{n}^{2}(\Omega)$.

Take $f \in L^{\infty}\left(\mathbb{R}^{+} ; H^{1}\right)$ such that $\widehat{f} \in L^{\infty}\left(\mathbb{R}^{+} ; H^{1}(\mathbb{R}) \cap H^{2}(\Omega)\right)$.

Then there exists $T^{\star}>0$ and a unique solution $\left(E, H_{e}\right)$ of (1.12) satisfying

$$
\begin{gathered}
\left(E, \hat{E}, H_{e}\right) \in L^{\infty}\left(\left[0, T^{\star}\left[; H^{1}(\mathbb{R})\right) \times L^{\infty}\left(\left[0, T^{\star}\left[; H^{2}(\Omega) \cap H^{1}(\mathbb{R})\right) \times L^{\infty}\left(\left[0, T^{\star}\left[; H_{n}^{2}(\Omega)\right),\right.\right.\right.\right.\right.\right. \\
\left(E, \hat{E}, H_{e}\right) \in C^{0}\left(\left[0, T^{\star}\left[; H^{1-\eta}(\mathbb{R})\right) \times C^{0}\left(\left[0, T^{\star}\left[; H^{2-\eta}(\Omega) \cap H^{1-\eta}(\mathbb{R})\right) \times C^{0}\left(\left[0, T^{\star}\left[; H^{2-\eta}(\Omega)\right), \quad \forall \eta>0 .\right.\right.\right.\right.\right.\right.
\end{gathered}
$$

Moreover if $\widehat{\nu}(0, \xi) \geq 0 \forall \xi \in \mathbb{R}$ then $\forall t \in\left[0, T^{\star}[, \widehat{\nu}(t, \xi) \geq 0\right.$.

The only case where we are able to prove a global existence result is the homogeneous case. From the physical point of view, it corresponds to a linear case where the pump wave has been cut off.

Theorem 1.2 (global solution in the homogeneous case). Let $E_{0}, H_{0}$, such that $\hat{E}_{0} \in H^{1}(\Omega) H_{0} \in H^{1}(\Omega)$. One moreover assume that

$$
\hat{\nu}_{e_{0}}(\xi)=\operatorname{sgn}(\xi) \partial_{\xi} H_{e 0}(\xi) \geq 0, \quad \forall \xi \in \Omega,
$$

and $\left(H_{0}+\xi^{2} \partial_{\xi}\left(|\xi|^{3}\left|\hat{E}_{0}\right|^{2}\right)\right)^{+} \in L^{\infty}(\Omega)$. Then there exists $E, H_{e}$ such that $\hat{E} \in L_{\text {loc }}^{\infty}\left(\mathbb{R}_{+}, H^{1}\right), H_{e} \in L_{\text {loc }}^{\infty}\left(\mathbb{R}_{+}, H^{1}\right)$ satisfying

$$
\begin{gathered}
i\left(\partial_{t} E+\nu * E\right)+\partial_{x}^{2} E=0 \\
\hat{\nu}(t, \xi)=\operatorname{sgn}(\xi) \partial_{\xi} H_{e}(t, \xi), \quad \forall \xi \in \Omega, \\
\partial_{t} H_{e}-\xi^{2} \partial_{\xi}\left(|\xi|^{3}|\hat{E}|^{2} \partial_{\xi} H_{e}\right)=0, \quad \forall \xi \in \Omega, \\
E(0, x)=E_{0}(x), \\
H_{e}(0, \xi)=H_{0}(\xi)
\end{gathered}
$$

and

$$
\partial_{\xi} H_{e}=0 \text { on } \partial \Omega
$$

in the weak sense.

Moreover $\forall t, \xi, \hat{\nu}(t, \xi) \geq 0$.

It follows that for all $t \in \mathbb{R}_{+}$,

$$
\int|E(t, x)|^{2} \mathrm{~d} x \leq \int\left|E_{0}(x)\right|^{2} \mathrm{~d} x
$$

The paper is organized as follows. In Section 2, we formally derive the nonlinear model (1.8)-(1.11). In Section 3, we introduce the dimensionless form of the system, and give the proofs of the main results. In Section 4 , we study a numerical scheme for (1.8)-(1.11) and present numerical results.

\section{Formal DeRIVATion OF THE SYSTEM}

The aim of this section is to present a formal derivation of system (1.8)-(1.11). For physical considerations, we refer to textbooks [5].

We consider here a plasma where collisions between the particles and the gravitational field are neglected. In this context, the Vlasov equation describes the evolution of the distribution function for each particle species 
$\alpha$ of the collision-less plasma in the phase space $(x, v)$ and time $t$. Denote by $q_{\alpha}$ the particle charge of specie $\alpha$ and by $m_{\alpha}$ the mass of specy $\alpha$. The Vlasov equation reads:

$$
\partial_{t} f_{\alpha}(x, v, t)+v \cdot \nabla_{x} f_{\alpha}(x, v, t)+\frac{q_{\alpha}}{m_{\alpha}}\left(E+\frac{1}{c} v \times B\right) \cdot \nabla_{v} f_{\alpha}(x, v, t)=0,
$$

$\alpha=e$ denotes the electrons and $\alpha=i$ the ions. The fields $E$ and $B$ correspond to the electric and magnetic fields respectively and are given by the Maxwell equations:

$$
\begin{aligned}
\nabla \times B & =\frac{4 \pi}{c} j+\frac{1}{c} \partial_{t} E, \\
\nabla \times E & =-\frac{1}{c} \partial_{t} B \\
\nabla . E & =4 \pi \rho, \\
\nabla . B & =0 .
\end{aligned}
$$

where

and

$$
\rho=-e\left(\int f_{e}(x, v, t) \mathrm{d} v-\int f_{i}(x, v, t) \mathrm{d} v\right)
$$

$$
j=-e\left(\int v f_{e}(x, v, t) \mathrm{d} v-\int v f_{i}(x, v, t) \mathrm{d} v\right)
$$

are the density of the total charge and total current respectively. The constant $c$ is the velocity of the light in the vacuum. Equations (2.2)-(2.3) yield:

$$
\frac{1}{c^{2}} \partial_{t}^{2} E+\nabla \times \nabla \times E=-\frac{4 \pi}{c^{2}} \partial_{t} j
$$

and since the mass of the electrons is very small compared to the mass of the ions $\left(m_{e} \ll m_{i}\right)$ and the Lorenz force is the same for electrons and ions, the contribution of the ions in the current $j$ can be neglected, so the electric field satisfies:

$$
\frac{1}{c^{2}} \partial_{t}^{2} E+\nabla \times \nabla \times E=\frac{4 \pi e}{c^{2}} \int v \partial_{t} f_{e} \mathrm{~d} v .
$$

In a situation where an electromagnetic wave is injected into a collision-less plasma, one can identify one highfrequency time scale for the evolution of the electronic plasma wave and the high-frequency electromagnetic wave. In nuclear fusion by inertial confinement, the time scale is the order $T=\frac{1}{\omega_{p e}}=10^{-15} \mathrm{~s}$ where $\omega_{p e}$ is the electron plasma frequency defined by $\omega_{p e}^{2}=\frac{4 \pi e^{2} n_{0}}{m_{e}}$ where $n_{0}$ is the background density of the homogeneous plasma.

\subsection{The high-frequency electron motion}

The goal of this part is to obtain an equation for the slowly varying amplitude $\mathcal{E}$ of the high frequency longitudinal electric field and its dependence on the slowly varying density fluctuation. The electric field is assumed to be decomposed as:

$$
E=\frac{1}{2}\left(\mathcal{E} \mathrm{e}^{-i \omega_{p e} t}+\text { c.c. }\right),
$$

and we make the time envelope approximation assuming $\partial_{t} \mathcal{E} \ll \omega_{p e} \mathcal{E}$. Here c.c. denotes the complex conjugate.

For any function $f$, we define its average over the fast time scale by

$$
\langle f\rangle=\frac{\omega_{p e}}{2 \pi} \int_{t}^{t+\frac{2 \pi}{\omega_{p e}}} f(x, s) \mathrm{d} s
$$


which gives the slowly varying components only.

With this definition, the slowly varying amplitude of the high-frequency component is given by

$$
\left\langle f \mathrm{e}^{i \omega_{p e} t}\right\rangle \mathrm{e}^{-i \omega_{p e} t} .
$$

Plugging (2.8) in (2.7) and taking the slowly varying amplitude of the resulting equations gives:

$$
\partial_{t}^{2} \mathcal{E}-2 i \omega_{p e} \partial_{t} \mathcal{E}-\omega_{p e}^{2} \mathcal{E}+c^{2} \nabla \times \nabla \times \mathcal{E}=4 \pi e\left\langle\mathrm{e}^{i \omega_{p e} t} \int v \partial_{t} f_{e}(x, v, t)\right\rangle .
$$

We therefore have to found the contribution of the right-hand side of (2.9), that is the contribution of $f_{e}$.

From now on, we work in the two dimension phase's space $(x, v)$. The distribution $f_{e}$ contains high and low frequency components, so that we can introduce the following decomposition:

$$
f_{e}(x, v, t)=f_{0}(x, v, t)+\frac{1}{2}\left(f_{1}(x, v, t) \mathrm{e}^{-i \omega_{p e} t}+c . c .\right),
$$

with $\partial_{t}\left(f_{0}, f_{1}\right) \ll \omega_{p e}\left(f_{0}, f_{1}\right)$.

Then plugging (2.10) in the right-hand side of (2.9) gives:

$$
\partial_{t}^{2} \mathcal{E}-2 i \omega_{p e} \partial_{t} \mathcal{E}-\omega_{p e}^{2} \mathcal{E}=-i \omega_{p e}\left(4 \pi e \int v f_{1}(x, v, t) \mathrm{d} v\right) .
$$

Now, we have to determine the contribution of the integral $\int v f_{1}(x, w, t) \mathrm{d} w$. To this aim, we have to think of the solution of the Vlasov equation under the form (2.10).

Plugging (2.10) in the Vlasov equation and keeping the low and high frequency components, we find that $\left(f_{0}, f_{1}\right)$ satisfy

$$
\begin{aligned}
& \partial_{t} f_{0}+v \partial_{x} f_{0}-\frac{e}{2 m_{e}}\left(\partial_{v} f_{1} \mathcal{E}^{*}+\partial_{v} f_{1}^{*} \mathcal{E}\right)=0, \\
& \partial_{t} f_{1}-i \omega_{p e} f_{1}+v \partial_{x} f_{1}=\frac{e}{m_{e}} \mathcal{E} \partial_{v} f_{0},
\end{aligned}
$$

and since $\partial_{t} f_{1} \ll \omega_{p e} f_{1}$, the following equation holds for $f_{1}$

$$
\left(-i \omega_{p e}+v \partial_{x}\right) f_{1}=\frac{e}{m_{e}} \mathcal{E} \partial_{v} f_{0} .
$$

Using (2.14), we found that $\mathcal{E}$ satisfies

$$
\partial_{t}^{2} \mathcal{E}-2 i \omega_{p e} \partial_{t} \mathcal{E}-\omega_{p e}^{2} \mathcal{E}=4 \pi e\left(\frac{e}{m_{e}} \mathcal{E} \int v \partial_{v} f_{0} \mathrm{~d} v-\partial_{x} \int v^{2} f_{1} \mathrm{~d} v\right)
$$

and denoting $\left\langle n_{e}\right\rangle=\int f_{0} \mathrm{~d} v$ the slowly varying electronic density over the slow time scale, the slowly varying amplitude $\mathcal{E}$ satisfies

$$
\partial_{t}^{2} \mathcal{E}-2 i \omega_{p e} \partial_{t} \mathcal{E}=\frac{\omega_{p e}^{2}}{n_{0}}\left(n_{0}-\left\langle n_{e}\right\rangle\right) \mathcal{E}-4 \pi e \partial_{x} \int v^{2} f_{1} \mathrm{~d} v .
$$

At this step, we have obtained three equations $(2.12),(2.13),(2.15)$ governing $\left(f_{0}, f_{1}, \mathcal{E}\right)$. The equation $(2.14)$ suggest that $f_{1}$ can be expressed in function of $f_{0}$ and then eliminated in $(2.12),(2.15)$. We now explain how to make this. 
In order to evaluate the contribution of $f_{1}$, we have to inverse the operator $\left(-i \omega_{p e}+v \partial_{x}\right)$ which symbol is an imaginary complex. To this aim, we add a small positive parameter $\varepsilon$ such that $f_{1}$ will be formally the limit of $f_{1}^{\varepsilon}$ solution of

$$
\varepsilon f_{1}^{\varepsilon}-i \omega_{p e} f_{1}^{\varepsilon}+v \partial_{x} f_{1}^{\varepsilon}=\alpha \mathcal{E} \partial_{v} f_{0}
$$

with $\alpha=\frac{e}{m_{e}}$. Using the fact that for any distribution $f$, we have $\widehat{\partial_{x} f}=i \xi \widehat{f}$, the solution of (2.16) can be written as

$$
f_{1}^{\varepsilon}=G^{\varepsilon} *_{x, v} S\left(f_{0}, \mathcal{E}\right)
$$

where $S\left(f_{0}, \mathcal{E}\right)$ denotes the operator $\alpha \mathcal{E} \partial_{v} f_{0}$ and $G^{\varepsilon}$ the Green's function associated with the equation (2.16)

$$
G^{\varepsilon}(x-y, v-w)=\frac{1}{2 i \pi} \int \mathrm{e}^{i \xi(x-y)} \frac{\delta(v-w)}{\xi \cdot v-\omega_{p e}-i \varepsilon} \mathrm{d} \xi .
$$

Before plugging (2.17) into (2.15) and taking the limit $\varepsilon$ goes to zero, we compute the moments $\int v^{p} f_{1}(x, v, t) \mathrm{d} v$ by using equation(2.14). We get:

$$
-i \omega_{p e} \int v^{p} f_{1} \mathrm{~d} v+\partial_{x} \int v^{p+1} f_{1} \mathrm{~d} v=\alpha \mathcal{E} \int v^{p} \partial_{v} f_{0} \mathrm{~d} v
$$

Using (2.18) for $p=2, p=3, p=4$ and using the symmetry properties of $f_{0}(v)$, one obtains

$$
\int v^{2} f_{1} \mathrm{~d} v=\frac{\alpha}{\omega_{p e}^{2}} \partial_{x}\left(\mathcal{E} \int v^{3} \partial_{v} f_{0} \mathrm{~d} v\right)+\frac{i}{\omega_{p e}^{3}} \partial_{x}^{3}\left(\int v^{5} f_{1} \mathrm{~d} v\right)
$$

or equivalently

$$
\int v^{2} f_{1} \mathrm{~d} v=\frac{\alpha}{\omega_{p e}^{2}} \partial_{x}\left(\mathcal{E} \int v^{3} \partial_{v} f_{0} \mathrm{~d} v\right)+\frac{i}{\omega_{p e}^{3}} \partial_{x}^{3}\left(\int v^{5} \lim _{\varepsilon \rightarrow 0^{+}} G^{\varepsilon} *_{x, v} S\left(f_{0}, \mathcal{E}\right) \mathrm{d} v\right) .
$$

Now to find the contribution of $f_{1}$ in the current we have to compute $-4 \pi e \partial_{x} \int v^{2} f_{1} \mathrm{~d} v$ in (2.15) thanks to (2.20).

For this purpose, we make the following Ansatz on $f_{0}$

$$
f_{0}(x, v, t)=F_{0}(v, t)+\delta f_{0}(x, v, t) .
$$

where $F_{0}$ is the spatially averaged distribution with $\left|\delta f_{0}\right| \ll\left|F_{0}\right|$. We also define, the electronic thermal velocity $v_{e}$ and the local fluctuation of velocity $\delta v_{e}(x, t)\left(\delta v_{e} \ll v_{e}\right)$ by

$$
n_{0} v_{e}^{2}=\int v^{2} F_{0} \mathrm{~d} v, n_{0} \delta v_{e}^{2}=\int v^{2} \delta f_{0} \mathrm{~d} v .
$$

After some integrations by parts in $v$, the term $-4 \pi e \partial_{x} \int v^{2} f_{1} \mathrm{~d} v$ exactly yields

$$
\begin{aligned}
-4 \pi e \partial_{x} \int v^{2} f_{1} \mathrm{~d} v= & 3 \frac{4 \pi e n_{0} v_{e}^{2} \alpha}{\omega_{p e}^{2}} \partial_{x}^{2} \mathcal{E}-\frac{4 \pi e i}{\omega_{p e}^{3}} \partial_{x}^{4}\left(\int v^{5} \lim _{\varepsilon \rightarrow 0^{+}} G^{\varepsilon} *_{x, v} S\left(F_{0}, \mathcal{E}\right) \mathrm{d} v\right) \\
& +3 \frac{4 \pi e n_{0} \alpha}{2 \omega_{p e}^{2}} \partial_{x}^{2}\left(\mathcal{E} \delta v_{e}^{2}\right)-\frac{4 \pi e i}{\omega_{p e}^{3}} \partial_{x}^{4}\left(\int v^{5} \lim _{\varepsilon \rightarrow 0^{+}} G^{\varepsilon} *_{x, v} S\left(\delta f_{0}, \mathcal{E}\right) \mathrm{d} v\right) \\
= & J_{1}+J_{2}+J_{3}+J_{4} .
\end{aligned}
$$

Using again the definitions of $\omega_{p e}$ and $\alpha$, the first term $J_{1}$ is given by

$$
J_{1}=3 v_{e}^{2} \partial_{x}^{2} \mathcal{E}
$$


Now, let us compute the second term $J_{2}$ of (2.22) accurately:

$$
\begin{aligned}
J_{2} & =-\frac{4 \pi e \alpha}{\omega_{p e}^{3}} \partial_{x}^{4}\left(\int v^{5} \lim _{\varepsilon \rightarrow 0^{+}} \iint \frac{1}{2 \pi} \mathrm{e}^{i \xi(x-y)} \frac{\partial_{v} F_{0}(v, t)}{\xi v-\omega_{p e}-i \varepsilon} \mathcal{E}(t, y) \mathrm{d} \xi \mathrm{d} y \mathrm{~d} v\right) \\
& =-\frac{4 \pi \mathrm{e}^{2}}{m_{e} \omega_{p e}^{3}} \int_{y} \mathcal{E}(t, y) \int_{\xi} \frac{1}{2 \pi} \xi^{4} \mathrm{e}^{i \xi(x-y)} I(t, \xi) \mathrm{d} \xi \mathrm{d} y,
\end{aligned}
$$

where

Some computations give

$$
I(t, \xi)=\lim _{\varepsilon \rightarrow 0^{+}} \int \frac{v^{5} \partial_{v} F_{0}(v, t)}{\xi v-\omega_{p e}-i \varepsilon} \mathrm{d} v .
$$

$$
I(t, \xi)=\mathcal{P} . \mathcal{V} \cdot\left(\frac{v^{5} \partial_{v} F_{0}(v, t)}{\xi v-\omega_{p e}}\right)+i \pi \operatorname{sgn}(\xi) \frac{\omega_{p e}^{5}}{\xi^{6}} \partial_{v} F_{0}\left(\frac{\omega_{p e}}{\xi}, t\right),
$$

where $\mathcal{P} . \mathcal{V}$. is the Cauchy Principal Value.

In [5], it is shown that the contribution of the first term of (2.25) can be neglected, we therefore obtain

$$
I(t, \xi)=i \pi \operatorname{sgn}(\xi) \frac{\omega_{p e}^{5}}{\xi^{6}} \partial_{v} F_{0}\left(\frac{\omega_{p e}}{\xi}, t\right) .
$$

Plugging (2.26) in (2.24) leads to

$$
\begin{aligned}
J_{2} & =-\frac{4 i \pi e^{2}}{m_{e} \omega_{p e}^{3}} \int_{y} \mathcal{E}(t, y) \int_{\xi} \frac{1}{2 \pi} \mathrm{e}^{i \xi(x-y)} \operatorname{sgn}(\xi) \frac{\omega_{p e}^{5}}{\xi^{2}} \partial_{v} F_{0}\left(\frac{\omega_{p e}}{\xi}, t\right) \mathrm{d} \xi \mathrm{d} y, \\
& =-2 i \frac{4 \pi e^{2} n_{0}}{m_{e} \omega_{p e}} \int_{y} \mathcal{E}(t, y) \int_{\xi} \frac{1}{2 \pi} \mathrm{e}^{i \xi(x-y)} \operatorname{sgn}(\xi) \frac{\pi \omega_{p e}^{3}}{2 n_{0} \xi^{2}} \partial_{v} F_{0}\left(\frac{\omega_{p e}}{\xi}, t\right) \mathrm{d} \xi \mathrm{d} y, \\
& =2 i \omega_{p e} \mathcal{E} * \nu(t, x),
\end{aligned}
$$

with

where

$$
\nu(t, x-y)=\frac{1}{2 \pi} \int_{\mathbb{R}} \mathrm{e}^{i \xi(x-y)} \widehat{\nu}(t, \xi) \mathrm{d} \xi,
$$

$$
\widehat{\nu}(t, \xi)=-\frac{\pi \omega_{p e}^{3}}{2 n_{0}|\xi| \xi} \partial_{v} F_{0}\left(t, \frac{\omega_{p e}}{\xi}\right) .
$$

Finally, neglecting the two last terms $J_{3}$ and $J_{4}$ (since $\delta f_{0} \ll F_{0}$ ), the slowly varying envelope satisfies the nonlinear Schrödinger equation

$$
2 i \omega_{p e} \partial_{t} \mathcal{E}+2 i \omega_{p e} \nu * \mathcal{E}+3 v_{e}^{2} \partial_{x}^{2} \mathcal{E}=\frac{\omega_{p e}^{2}}{n_{0}}\left(\left\langle n_{e}\right\rangle-n_{0}\right) \mathcal{E} .
$$

In order to close the system, we have to find an equation evolving $F_{0}$ and an equation evolving the low-frequency $\delta n_{e}=\left\langle n_{e}\right\rangle-n_{0}$.

The equation involving the spatially averaged distribution $F_{0}$ is recovered by substituting the function (2.17) solution of (2.16) into the right-hand side of (2.12), taking the spatial average and let $\varepsilon$ tends to zero.

In this paper, we only give a sketch of the computations and show what happens with the first non-linear term of (2.12) $N L=\lim _{\varepsilon \rightarrow 0^{+}} \partial_{v} f_{1}^{\varepsilon} \mathcal{E}^{*}$. For more details, see [16]. 
Plugging (2.17) in $N L$, using the definition of $G^{\varepsilon}$, taking the spatial average of $N L$ and let $\varepsilon$ tends to zero yields

$$
\begin{aligned}
\int N L(t, x, v) \mathrm{d} x & =\alpha \partial_{v} \iint_{x, y} \lim _{\varepsilon \rightarrow 0^{+}} \int_{\xi} \frac{1}{2 i \pi} \mathrm{e}^{i \xi(x-y)} \frac{\partial_{v} F_{0}(v, t)}{\xi v-\omega_{p e}-i \varepsilon} \mathcal{E}(t, y) \mathcal{E}^{*}(t, x) \mathrm{d} \xi \mathrm{d} y \mathrm{~d} x \\
& =\alpha \partial_{v}\left(\partial_{v} F_{0}(v, t) \iint_{x, y} \frac{1}{2 i \pi} \mathcal{E}(t, y) \mathcal{E}^{*}(t, x) \frac{i \pi}{|v|} \mathrm{e}^{i \xi^{*}(x-y)} \mathrm{d} y \mathrm{~d} x\right)
\end{aligned}
$$

with $\xi^{*}=\frac{\omega_{p e}}{v}$. Using the Fubini's theorem, one gets

$$
\begin{aligned}
\int N L(t, x, v) \mathrm{d} x & =\frac{\alpha}{2} \partial_{v}\left(\frac{1}{|v|} \partial_{v} F_{0}(v, t) \iint_{x, y} \mathrm{e}^{i \xi^{*}(x-y)} \mathcal{E}(t, y) \mathcal{E}^{*}(t, x) \mathrm{d} y \mathrm{~d} x\right) \\
& =\frac{\alpha}{2} \partial_{v}\left(\frac{1}{|v|} \partial_{v} F_{0}(v, t) \int \mathrm{e}^{i \xi^{*} x} \mathcal{E}^{*}(t, x) \mathrm{d} x \int \mathrm{e}^{-i \xi^{*} y} \mathcal{E}(t, y) \mathrm{d} y\right) \\
& =\frac{\alpha}{2} \partial_{v}\left(\frac{1}{|v|}\left|\widehat{\mathcal{E}}\left(t, \xi^{*}\right)\right|^{2} \partial_{v} F_{0}(v, t)\right)
\end{aligned}
$$

Finally, we find the usual quasi-linear diffusion equation

$$
\partial_{t} F_{0}-\partial_{v}\left(D(t, v) \partial_{v} F_{0}\right)=0, \text { with } D(t, v)=\frac{e^{2}}{2 m_{e}^{2}|v|}\left|\widehat{\mathcal{E}}\left(t, \frac{\omega_{p e}}{v}\right)\right|^{2}
$$

where the diffusion coefficient $D(t, v)$ depends on the spectral density energy $\left|\widehat{\mathcal{E}}\left(\frac{\omega_{p e}}{v}\right)\right|^{2}$.

As usual, the plasma is assumed to be quasi-neutral on the slow ion acoustic time scale, that is $\delta n_{e}=\delta n$ where $\delta n$ is the fluctuation of the ion density.

Now we look for an equation involving $\delta n_{i}$. Since $m_{i} \gg m_{e}$, the study is much simpler and we assume that the ion distribution function is Maxwellian.

In this context, one can see in [3], [15], [20] and [21], that $\delta n_{i}$ satisfies the wave equation

$$
\partial_{t}^{2} \delta n-c_{s}^{2} \partial_{x}^{2} \delta n=\frac{1}{16 \pi m_{i}} \partial_{x}^{2}\left(|\mathcal{E}|^{2}\right)
$$

where $c_{s}=\sqrt{\frac{T_{e}}{m_{i}}}$ is the ion acoustic velocity.

Finally, we have to deal with the following system

$$
\begin{aligned}
& 2 i \omega_{p e}\left(\partial_{t} \mathcal{E}+\nu * \mathcal{E}\right)+3 v_{e}^{2} \partial_{x}^{2} \mathcal{E}=\frac{\omega_{p e}^{2}}{n_{0}} \delta n \mathcal{E}, \\
& \partial_{t}^{2} \delta n-c_{s}^{2} \partial_{x}^{2} \delta n=\frac{1}{16 \pi m_{i}} \partial_{x}^{2}\left(|\mathcal{E}|^{2}\right), \\
& \widehat{\nu}(\xi, t)=-\frac{\pi \omega_{p e}^{3}}{2 \xi n_{0}|\xi|} \partial_{v} F_{e}\left(\frac{\omega_{p e}}{\xi}\right), \\
& \partial_{t} F_{0}=\partial_{v}\left(D(v, t) \partial_{v} F_{0}\right), \quad D(v, t)=\frac{e^{2}}{2 m_{e}^{2}|v|}\left|\widehat{\mathcal{E}}\left(\xi=\frac{\omega_{p e}}{v}, t\right)\right|^{2} .
\end{aligned}
$$


It describes the interaction between the high-frequency envelope of the longitudinal electric field and the lowfrequency density fluctuation (wave-wave process) and the resonant interaction between the electrons and the longitudinal electron plasma waves (wave-particle process).

Usually the electron plasma waves are created by a pump wave obtained by the stimulated Raman scattering, acting as a source term in (2.32) (see [3]). However, it is possible to add a given pump wave in the derivation of the Schrödinger equation by changing the envelope approximation (2.8) by

$$
E(x, t)=\frac{1}{2}\left(E_{p}(t, x) \mathrm{e}^{i(k x-\omega t)}+\mathcal{E}(t, x)\right) \mathrm{e}^{-i \omega_{p e} t}+\text { c.c. },
$$

and we can replace $(2.32)$ with

$$
2 i \omega_{p e}\left(\partial_{t} \mathcal{E}+\nu * \mathcal{E}\right)+3 v_{e}^{2} \partial_{x}^{2} \mathcal{E}=\frac{\omega_{p e}^{2}}{n_{0}} \delta n \mathcal{E}+\omega_{p e}^{2} E_{p} \mathrm{e}^{i\left(k_{1} x-\omega_{1} t\right)} .
$$

Since the dispersion relation of the linear part of $(2.36)$ is $\omega=\frac{3 v_{e}^{2} k^{2}}{2 \omega_{p e}}$, we choose $\left(k_{1}, \omega_{1}\right)$ satisfying this relation.

\subsection{Dimensionless form}

We now introduce a dimensionless form of (2.32)-(2.35).

We use $T=\frac{1}{\omega_{p e}}$ as time scale and $L=\lambda_{D e}$ as space scale (where $\lambda_{D e}=\frac{v_{e}}{\omega_{p e}}$ is the Debye's length) and introduce

$$
\begin{aligned}
& \widetilde{E}=\frac{e}{m_{e} v_{e} \omega_{p e}} \mathcal{E}, \quad \widetilde{\widehat{\nu}}=\frac{1}{\omega_{p e}} \widehat{\nu}, \\
& \widetilde{k_{1}}=k_{1} \lambda_{D e}, \quad \widetilde{\omega_{1}}=\frac{\omega_{1}}{\omega_{p e}}, \\
& \widetilde{F_{0}}=\frac{v_{e}}{n_{0}} F_{0}, \quad \widetilde{\delta n}=\frac{1}{n_{0}} \delta n .
\end{aligned}
$$

Dropping the tildes, we get the following system:

$$
\begin{aligned}
& 2 i\left(\partial_{t} E+\nu * E\right)+3 \partial_{x}^{2} E=\delta n E+E_{p}(x) \mathrm{e}^{i\left(k_{1} x-\omega_{1} t\right)}, \\
& \partial_{t}^{2} \delta n-\mu \partial_{x}^{2} \delta n=\frac{\mu}{4} \partial_{x}^{2}\left(|E|^{2}\right), \\
& \widehat{\nu}(t, \xi)=-\frac{\pi}{2 \xi|\xi|} \partial_{v} F_{e}\left(t, \frac{1}{\xi}\right), \\
& \partial_{t} F_{e}=\partial_{v}\left(D(t, v) \partial_{v} F_{e}\right), D(t, v)=\frac{1}{4|v|}\left|\widehat{E}\left(t, \frac{1}{v}\right)\right|^{2},
\end{aligned}
$$

where $\mu=\frac{c_{s}^{2}}{v_{e}^{2}}=\frac{m_{e}}{m_{i}}$.

Usually, (2.37)-(2.38) are satisfied on the whole space $\mathbb{R}$. But (2.39)-(2.40) are valid only for bounded speeds and far away from zero. The velocity domain that we consider is

$$
v \in \Omega_{v}=[-A,-a] \cup[a, A], \quad(A>a>0),
$$

which gives frequency domain of the form,

$$
\xi \in \Omega_{\xi}=\left[-a^{-1},-A^{-1}\right] \cup\left[A^{-1}, a^{-1}\right], \quad(A>a>0) .
$$

Apart from this set, $\hat{\nu}_{e}(., \xi)$ is extended to 0 . 
Moreover, in order to study the system (2.37)-(2.40), it is more convenient to write it by using the variable $\xi$ rather than the variable $v$ on equations (2.39)-(2.40). Then denoting

$$
H_{e}(t, \xi)=F_{0}\left(t, \frac{1}{\xi}\right),
$$

the system that we study is (we set all coefficients equal to one excepted $\mu$ )

$$
\begin{gathered}
i\left(\partial_{t} E+\nu * E\right)+\partial_{x}^{2} E=\delta n E+E_{p}(x) \mathrm{e}^{i\left(k_{1} x-\omega_{1} t\right)}, \quad x \in \mathbb{R}, t \geq 0, \\
\frac{1}{\mu} \partial_{t}^{2} \delta n-\partial_{x}^{2} \delta n=\partial_{x}^{2}\left(|E|^{2}\right), \quad x \in \mathbb{R}, t \geq 0, \\
\partial_{t} H_{e}-\xi^{2} \partial_{\xi}\left(|\xi|^{3}|\hat{E}(t, \xi)|^{2} \partial_{\xi} H_{e}\right)=0, \quad \xi \in \Omega_{\xi}, t \geq 0, \\
\hat{\nu}(t, \xi)=\operatorname{sgn}(\xi) \partial_{\xi} H_{e}(t, \xi) 1_{\Omega_{\xi}} .
\end{gathered}
$$

The boundary conditions are

$$
\partial_{\xi} H_{e \mid \partial \Omega_{\xi}}=0, \quad \hat{\nu}_{\mid \partial \Omega_{\xi}}=0
$$

\section{Proofs of the MAIN RESUlts}

Let us consider the system (2.41)-(2.42). In the following, we will denote $\Omega_{\xi}$ by $\Omega$. Unfortunately, we are not able to handle the general case. So we will consider only the case $\mu=+\infty$ corresponding to a subsonic regime. Therefore, the system (2.41)-(2.42) to be solved becomes

$$
\begin{aligned}
& i\left(\partial_{t} E+\nu * E\right)+\partial_{x}^{2} E=-|E|^{2} E+S(t, x), \\
& \partial_{t} H_{e}-\xi^{2} \partial_{\xi}\left(|\xi|^{3}|\hat{E}|^{2} \partial_{\xi} H_{e}\right)=0, \quad \forall \xi \in \Omega, \\
& \partial_{\xi} H_{e \mid \Omega}=0, \\
& \hat{\nu}(\xi, .)=\operatorname{sgn}(\xi) \partial_{\xi} H_{e} 1_{\Omega}, \\
& H_{e}(., 0)=H_{e 0}(.), E(., 0)=E_{0}(.) .
\end{aligned}
$$

The functional space for $H_{e}$ is

$$
H_{n}^{2}(\Omega)=\left\{u \in H^{2}(\Omega) \text { such that } \partial_{n} u_{\mid \partial \Omega}=0\right\} .
$$

In order to simplify computations, and without loss of generality, we take $\Omega=]-2,-1[\cup] 1,2[$.

The first step of the proof is the construction of solutions given by Theorem 1.1 for a regularized problem. The regularization is obtained by taking a non degenerate dissipation on equation (2.42), namely one replaces $|\xi|^{3}|\hat{E}|^{2}$ by $|\xi|^{3}|\hat{E}|^{2}+\varepsilon$ with $\varepsilon>0$.

Moreover the left hand side of the first equation of (3.1) is replaced by a source term $f$. One then obtains local existence for solutions whose time of existence depends on $\varepsilon$ and $f$. We obtain a solution satisfying

$$
\left(E, \hat{E}, H_{e}\right) \in L^{\infty}\left(0, T^{\star} ; H^{1}(\mathbb{R})\right) \times L^{\infty}\left(0, T^{\star} ; H^{2}(\Omega) \cap H^{1}(\mathbb{R})\right) \times L^{\infty}\left(0, T^{\star} ; H_{n}^{2}(\Omega)\right) .
$$

The next step consists in replacing $f$ by $|E|^{2} E$ by using a fixed point method. The important point is that $H^{1}$ is an algebra and $E, \hat{E}$ are in $H^{1}$ so that

$$
\widehat{|E|^{2} E} \in W^{2, \infty}(\mathbb{R})
$$

and therefore

$$
\widehat{|E|^{2} E} \in H^{2}(\Omega) .
$$


The last step consists in obtaining estimates that are uniform with respect to $\varepsilon$. In physical situation the term $\nu * E$ is a damping term. The positivity of $\widehat{\nu}(t, \xi)$ is then obtained by using the maximum principle on equation (2.39). In order to prove Theorem 1.2, we use an algebraic cancellation between the left hand side of (2.37) and the dissipative part of (2.40). We now prove both theorems.

\subsection{Local existence of weak solutions}

In order to prove Theorems 1.1 and 1.2, we consider a problem with non degenerate dissipation on the diffusion equation.

\subsubsection{Non degenerated simplified problem}

For $\varepsilon>0$, we consider

$$
\begin{aligned}
& i\left(\partial_{t} E^{\varepsilon}+\nu^{\varepsilon} * E^{\varepsilon}\right)+\partial_{x}^{2} E^{\varepsilon}=f, \\
& \partial_{t} H_{e}^{\varepsilon}-\xi^{2} \partial_{\xi}\left(\left(|\xi|^{3}|\hat{E}|^{2}+\varepsilon\right) \partial_{\xi} H_{e}^{\varepsilon}\right)=0, \quad \forall \xi \in \Omega, \\
& \partial_{\xi} H_{e}^{\varepsilon}=0 \\
& \hat{\nu}_{e}^{\varepsilon}=\operatorname{sgn}(\xi) \partial_{\xi} H_{e}^{\varepsilon} 1_{\Omega} \\
& H_{e}^{\varepsilon}(0, .)=H_{e 0}(.), E^{\varepsilon}(0, .)=E_{0}(.),
\end{aligned}
$$

with $f \in L^{\infty}\left(0, T ; H^{1}(\mathbb{R})\right), \hat{f} \in L^{\infty}\left(0, T ; H^{2}(\Omega) \cap H^{1}(\mathbb{R})\right)$.

Proposition 3.1. Let $E_{0} \in H^{1}(\mathbb{R})$ such that $\hat{E}_{0} \in H^{1}(\mathbb{R})$, and let $H_{e 0} \in H^{1}(\Omega)$, then there exist $T^{\star}>0$ and a unique solution $\left(E^{\varepsilon}, H_{e}^{\varepsilon}\right)$ of $(3.2)$ such that

$$
\left(E^{\varepsilon}, \hat{E}^{\varepsilon}, H_{e}^{\varepsilon}\right) \in C^{0}\left(\left[0, T^{\star}\left[; H^{1}(\mathbb{R})\right) \times C^{0}\left(\left[0, T^{\star}\left[; H^{1}(\mathbb{R})\right) \times C^{0}\left(\left[0, T^{\star}\left[; H^{1}(\Omega)\right) .\right.\right.\right.\right.\right.\right.
$$

If moreover $\hat{E}_{0} \in H^{2}(\Omega) \cap H^{1}(\mathbb{R})$, and $H_{e 0} \in H_{n}^{2}(\Omega)$ then

$$
\left(E^{\varepsilon}, \hat{E}^{\varepsilon}, H_{e}^{\varepsilon}\right) \in C^{0}\left(\left[0, T^{\star}\left[; H^{1}(\mathbb{R})\right) \times C^{0}\left(\left[0, T^{\star}\left[; H^{2}(\Omega) \cap H^{1}(\mathbb{R})\right) \times C^{0}\left(\left[0, T^{\star}\left[; H_{n}^{2}(\Omega)\right) .\right.\right.\right.\right.\right.\right.
$$

The proof of this proposition is obtained with a fixed point method.

Let us consider a function $G_{e}:(x, t) \in \mathbb{R} \times \mathbb{R}^{+} \rightarrow \mathbb{R}$ such that $G_{e} \in L^{\infty}\left(\mathbb{R}^{+}, H_{n}^{2}(\Omega)\right)$, and define $\mu_{e}=\operatorname{sgn}(\xi) \partial_{\xi} G_{e} 1_{\Omega}$. Consider $E^{\varepsilon}, H^{\varepsilon}$ and $\nu^{\varepsilon}$ solution of the following system,

$$
\begin{aligned}
& i\left(\partial_{t} E^{\varepsilon}+\mu_{e} * E^{\varepsilon}\right)+\partial_{x}^{2} E^{\varepsilon}=f, \\
& \partial_{t} H_{e}^{\varepsilon}-\xi^{2} \partial_{\xi}\left(\left(|\xi|^{3}|\hat{E}|^{2}+\varepsilon\right) \partial_{\xi} H_{e}^{\varepsilon}\right)=0, \quad \forall \xi \in \Omega, \\
& \partial_{\xi} H_{e \mid \Omega}^{\varepsilon}=0 \\
& \hat{\nu}_{e}^{\varepsilon}=\operatorname{sgn}(\xi) \partial_{\xi} H_{e}^{\varepsilon} 1_{\Omega} \\
& H_{e}^{\varepsilon}(., 0)=H_{e 0}(.), E(., 0)=E_{0}(.) .
\end{aligned}
$$

We want to show that the map $\tau: G_{e} \mapsto H_{e}^{\varepsilon}$ is a contraction on a suitable metric spaces.

For any $R>0$, we denote by $B_{R}(H)$ the ball of the space $H$ centered on 0 with radius $R$.

For $R$ large enough and $T$ small enough, we show that $\tau$ maps

$$
B_{R}\left(L^{\infty}\left(0, T ; H^{1}(\mathbb{R}) \cap \mathcal{F}\left(H^{1}(\mathbb{R})\right) \cap H_{n}^{2}(\Omega)\right) \cap L^{2}\left(0, T, H_{n}^{3}(\Omega)\right)\right)
$$

into itself and maps

$$
B_{R}\left(L^{\infty}\left(0, T ; H^{1}(\mathbb{R}) \cap \mathcal{F}\left(H^{1}(\mathbb{R})\right)\right) \cap L^{2}\left(0, T, H_{n}^{2}(\Omega)\right)\right)
$$

into itself. 
Let us prove this last result.

Performing an $L^{2}$ estimate on the first and second equation of (3.3) yields

$$
\begin{aligned}
\frac{\mathrm{d}}{\mathrm{d} t}|\hat{E}|_{L^{2}(\mathbb{R})}^{2} & \leq 2 \int_{\Omega}\left|\hat{\mu}_{e}\right||\hat{E}|^{2}+2 \int_{\mathbb{R}}|\hat{f}||\hat{E}| \\
& \leq c\left|\hat{\mu}_{e}\right|_{H^{1}(\Omega)}|\hat{E}|_{L^{2}(\Omega)}^{2}+|\hat{f}|_{L^{2}(\mathbb{R})}^{2}+|\hat{E}|_{L^{2}(\mathbb{R})}^{2}
\end{aligned}
$$

and

$$
\frac{\mathrm{d}}{\mathrm{d} t}\left|\xi^{-1} H_{e}^{\varepsilon}\right|_{L^{2}(\Omega)}^{2}+2 \int_{\Omega}\left(|\xi|^{3}|\hat{E}|^{2}+\varepsilon\right)\left|\partial_{\xi} H_{e}^{\varepsilon}\right|^{2}=0 .
$$

We now perform an $H^{1}$ estimate in the $\xi$ space

$$
\frac{\mathrm{d}}{\mathrm{d} t}\left|\partial_{\xi} \hat{E}\right|_{L^{2}(\mathbb{R})}^{2}+2 \int_{\Omega} \hat{\mu}_{e}\left|\partial_{\xi} \hat{E}\right|^{2}+2 \Im\left(\int_{\Omega} \partial_{\xi} \hat{\mu}_{e} \hat{E} \partial_{\xi} \hat{\hat{E}}\right)=2 \Im\left(\int_{\mathbb{R}} \partial_{\xi} \hat{f} \partial_{\xi} \hat{\hat{E}}\right)+4 \Im\left(\int_{\mathbb{R}} \xi \hat{E} \partial_{\xi} \hat{\hat{E}}\right)
$$

This gives,

$$
\frac{\mathrm{d}}{\mathrm{d} t}\left|\partial_{\xi} \hat{E}\right|_{L^{2}(\mathbb{R})}^{2} \leq c\left|\hat{\mu}_{e}\right|_{H^{1}(\Omega)}|\hat{E}|_{H^{1}(\Omega)}^{2}+\left|\partial_{\xi} \hat{f}\right|_{L^{2}(\mathbb{R})}^{2}+2\left|\partial_{\xi} \hat{E}\right|_{L^{2}(\mathbb{R})}^{2}+C|\hat{E}|_{H^{1}(\mathbb{R})}^{2}+c|E|_{H^{1}(\mathbb{R})}^{2}
$$

An $H^{1}$ estimate on $H_{e}^{\varepsilon}$ leads to

$$
\frac{\mathrm{d}}{\mathrm{d} t}\left|\partial_{\xi} H_{e}^{\varepsilon}\right|_{L^{2}(\Omega)}^{2}+2 \int_{\Omega} \xi^{2}\left(|\xi|^{3}|\hat{E}|^{2}+\varepsilon\right)\left|\partial_{\xi}^{2} H_{e}^{\varepsilon}\right|^{2} \leq \varepsilon\left|\partial_{\xi} H_{e}^{\varepsilon}\right|_{L^{2}(\Omega)}^{2}+\varepsilon^{-1}\left|\xi^{2} \partial_{\xi}\left(|\xi|^{3}|\hat{E}|^{2}\right)\right|_{L^{1}(\Omega)} .
$$

Summing theses estimates, it follows that,

$$
\begin{aligned}
\frac{\mathrm{d}}{\mathrm{d} t}\left(|\hat{E}|_{H^{1}(\mathbb{R})}^{2}+\left|\xi^{-1} H_{e}^{\varepsilon}\right|_{L^{2}(\Omega)}^{2}+\left|\xi^{-1} \partial_{\xi} H_{e}^{\varepsilon}\right|_{L^{2}(\Omega)}^{2}\right)+\int_{\Omega}\left(|\xi|^{3}|\hat{E}|+\varepsilon\right)\left(\xi^{2}\left|\partial_{\xi} H_{e}^{\varepsilon}\right|^{2}+\left|H_{e}^{\varepsilon}\right|^{2}\right) & \leq c\left(1+\varepsilon^{-1}+\left|\hat{\mu}_{e}\right|_{H^{1}}\right)|\hat{E}|_{H^{1}(\mathbb{R})}^{2}+|\hat{f}|_{H^{1}(\Omega)}^{2}+c|E|_{H^{1}(\mathbb{R})}^{2}
\end{aligned}
$$

We still have to obtain an $H^{1}$ estimate for $E^{\varepsilon}$ in the $x$ space. We apply $\partial_{x}$ on the first equation of (3.3), multiplying by $\partial_{x} \bar{E}$ and take the imaginary part gives:

$$
\frac{\mathrm{d}}{\mathrm{d} t} \int\left|\partial_{x} E\right|^{2} \leq 2\left|\hat{\mu}_{e}\right|_{L^{\infty}}\left|\partial_{x} E\right|_{L^{2}}^{2}+2\left|\partial_{x} f\right|_{L^{2}}\left|\partial_{x} E\right|_{L^{2}}
$$

That leads to

$$
\frac{\mathrm{d}}{\mathrm{d} t} \int\left|\partial_{x} E\right|^{2} \leq\left(2\left|\hat{\mu}_{e}\right|_{L^{\infty}}+1\right)\left|\partial_{x} E\right|_{L^{2}}^{2}+\left|\partial_{x} f\right|_{L^{2}}^{2}
$$

Thanks to the non degenerate diffusion term, we recover the estimate of $\hat{\nu}$ in $L^{2}\left(0, T ; H^{1}(\Omega)\right)$. By choosing $T$ small enough (depending on $\varepsilon$ ) and $R$ large enough (depending on the size of initial data), the map $\tau$ which associated $G_{e}$ with $H_{e}^{\varepsilon}$ maps

$$
B_{R}\left(L^{\infty}\left(0, T ; H^{1}(\mathbb{R}) \cap \mathcal{F}\left(H^{1}(\mathbb{R})\right)\right) \cap L^{2}\left(0, T, H_{n}^{2}(\Omega)\right)\right)
$$

into itself.

The contraction property is obtained in $L^{\infty}\left(0, T ; L^{2}(\mathbb{R}) \cap \mathcal{F}\left(H^{1}(\mathbb{R})\right)\right) \cap L^{2}\left(0, T, H^{1}(\Omega)\right)$. Let $G_{e}^{1}$, $G_{e}^{2}$ belonging to $B_{R}\left(L^{\infty}\left(0, T ; H^{1}(\mathbb{R}) \cap \mathcal{F}\left(H^{1}(\mathbb{R})\right)\right) \cap L^{2}\left(0, T, H_{n}^{2}(\Omega)\right)\right)$. Denote by $\left(E^{1}, H_{e}^{\varepsilon, 1}, \nu^{\varepsilon, 1}\right),\left(E^{2}, H_{e}^{\varepsilon, 2}, \nu^{\varepsilon, 2}\right)$ 
the solutions of (3.3) associated with $G_{e}^{1}$ and $G_{e}^{2}$ respectively. Denote by $E=E^{1}-E^{2}, H_{e}^{\varepsilon}=H_{e}^{\varepsilon, 1}-H_{e}^{\varepsilon, 2}$, $\nu_{e}^{\varepsilon}=\nu^{\varepsilon, 1}-\nu^{\varepsilon, 2}$. Then for all $\eta>0$, we have,

$$
\frac{\mathrm{d}}{\mathrm{d} t}|\hat{E}|_{L^{2}(\Omega)}^{2} \leq K(\eta, R)|\hat{E}|_{L^{2}(\Omega)}^{2}+\eta\left|\mu_{e}\right|_{L^{2}(\Omega)}^{2}
$$

where $K$ depends of $\eta$ and $R$.

$$
\frac{\mathrm{d}}{\mathrm{d} t}\left|H_{e}^{\varepsilon}\right|_{L^{2}(\Omega)}^{2}+\int_{\Omega}\left(\left|\hat{E^{1}}\right|^{2}+\varepsilon\right)\left|\partial_{\xi} H_{e}^{\varepsilon}\right|^{2} \leq C(R, \varepsilon)|\hat{E}|_{L^{2}(\Omega)}^{2}
$$

According to Gronwall's lemma, we easily conclude that

$$
\left|H_{e}^{\varepsilon}\right|_{L^{\infty}\left(0, T ; L^{2}(\Omega)\right)}^{2}+\varepsilon\left|\partial_{\xi} H_{e}^{\varepsilon}\right|_{L^{2}\left(0, T ; L^{2}(\Omega)\right)}^{2} \leq C(R, \varepsilon) \exp (K T) \eta\left|\mu_{e}\right|_{L^{2}\left(0, T ; L^{2}(\Omega)\right)}^{2}
$$

The contraction property is obtained by choosing $\eta$ and $T$ small enough.

\subsubsection{The complete non degenerated problem}

For $\varepsilon>0$, we consider

$$
\begin{aligned}
& i\left(\partial_{t} E^{\varepsilon}+\nu^{\varepsilon} * E^{\varepsilon}\right)+\partial_{x}^{2} E^{\varepsilon}=-\left|E^{\varepsilon}\right|^{2} E^{\varepsilon}+S, \\
& \partial_{t} H_{e}^{\varepsilon}-\xi^{2} \partial_{\xi}\left(\left(|\xi|^{3}\left|\hat{E}^{\varepsilon}\right|^{2}+\varepsilon\right) \partial_{\xi} H_{e}^{\varepsilon}\right)=0, \quad \forall \xi \in \Omega, \\
& \partial_{\xi} H_{e \mid \Omega}^{\varepsilon}=0, \\
& \hat{\nu}^{\varepsilon}(., \xi)=\operatorname{sgn}(\xi) \partial_{\xi} H_{e}^{\varepsilon} 1_{\Omega}, \\
& H_{e}^{\varepsilon}(., 0)=H_{e 0}(.), E^{\varepsilon}(., 0)=E_{0}(.) .
\end{aligned}
$$

Proposition 3.2. Let $S \in C^{0}\left(\mathbb{R}^{+} ; H^{1}(\mathbb{R})\right)$ such that $\hat{S} \in C^{0}\left(\mathbb{R}^{+} ; W^{2, \infty}(\mathbb{R})\right)$. Let $E_{0} \in H^{1}(\mathbb{R})$ such that $\hat{E}_{0} \in H^{1}(\mathbb{R})$, and let $H_{e 0} \in H^{1}(\Omega)$. Then there exists $T^{\star}>0$ and a unique solution $\left(E^{\varepsilon}, H_{e}^{\varepsilon}\right)$ of $(3.4)$ such that

$$
\left(E^{\varepsilon}, \hat{E}^{\varepsilon}, H_{e}^{\varepsilon}\right) \in C^{0}\left(0, T^{\star} ; H^{1}(\mathbb{R})\right) \times C^{0}\left(0, T^{\star} ; H^{1}(\mathbb{R})\right) \times C^{0}\left(0, T^{\star} ; H^{1}(\Omega)\right) .
$$

If moreover $\hat{E}_{0} \in H^{1}(\mathbb{R}) \cap H^{2}(\Omega)$, and $H_{e 0} \in H_{n}^{2}(\Omega)$, one has

$$
\left(E^{\varepsilon}, \hat{E}^{\varepsilon}, H_{e}^{\varepsilon}\right) \in C^{0}\left(0, T^{\star} ; H^{1}(\mathbb{R})\right) \times C^{0}\left(0, T^{\star} ; H^{1}(\mathbb{R}) \cap H^{2}(\Omega)\right) \times C^{0}\left(0, T^{\star} ; H_{n}^{2}(\Omega)\right) .
$$

According to Proposition 3.1, problem (3.2) has a solution. Denote by $f=|D|^{2} D+S$ where $D$ belongs to $C^{0}\left(\left[0, T^{\star}\left[, H^{1}(\mathbb{R})\right)\right.\right.$ and $\hat{D}$ belongs to $C^{0}\left(\left[0, T^{\star}\left[, H^{1}(\mathbb{R})\right)\right.\right.$. It follows that $f$ is in $C^{0}\left(\left[0, T^{\star}\left[, H^{1}(\mathbb{R})\right)\right.\right.$ because $H^{1}(\mathbb{R})$ is an algebra in one dimension and moreover $\hat{f}=\hat{D} \star \hat{D} \star \hat{D}+\hat{S}$ belongs to $C^{0}\left(\left[0, T^{\star}\left[; H^{1}(\mathbb{R})\right)\right.\right.$ because $\hat{D}$ is in $C^{0}\left(\left[0, T^{\star}\left[; L^{1}(\mathbb{R})\right)\right.\right.$.

In the same way,

$$
\partial_{\xi}^{2} \hat{f}=\hat{D} \star \partial_{\xi} \hat{\bar{D}} \star \partial_{\xi} \hat{D}+\partial_{\xi}^{2} \hat{S}
$$

belongs to $C^{0}\left(\left[0, T^{\star}\left[; L^{\infty}(\mathbb{R})\right)\right.\right.$ resulting from the convolution between a $L^{1}(\mathbb{R})$-function and two $L^{2}(\mathbb{R})$-functions. 
We will show the above proposition by using a fixed point method on the map $\tau$ which associates $D$ with $E^{\varepsilon}, H_{e}^{\varepsilon}$ and $\nu^{\varepsilon}$ solutions of

$$
\begin{aligned}
& i\left(\partial_{t} E^{\varepsilon}+\nu^{\varepsilon} * E^{\varepsilon}\right)+\partial_{x}^{2} E^{\varepsilon}=-|D|^{2} D+S, \\
& \partial_{t} H_{e}^{\varepsilon}-\xi^{2} \partial_{\xi}\left(\left(|\xi|^{3}\left|\hat{E}^{\varepsilon}\right|^{2}+\varepsilon\right) \partial_{\xi} H_{e}^{\varepsilon}\right)=0, \quad \forall \xi \in \Omega, \\
& \partial_{\xi} H_{e}^{\varepsilon} \mid \Omega=0, \\
& \hat{\nu}^{\varepsilon}(., \xi)=\operatorname{sgn}(\xi) \partial_{\xi} H_{e}^{\varepsilon} 1_{\Omega}, \\
& H_{e}^{\varepsilon}(., 0)=H_{e 0}(.), E^{\varepsilon}(., 0)=E_{0}(.) .
\end{aligned}
$$

If $H_{e 0} \in H^{1}(\Omega), E_{0} \in H^{1}(\mathbb{R})$ and $\hat{E}_{0} \in H^{1}(\mathbb{R})$, we show that $\tau$ maps $B_{R}\left(H_{T}\right)$ into itself, for $R$ large enough and $T$ small enough, where $H_{T}$ is

$$
H_{T}=\left\{e \in L^{\infty}\left(0, T ; H^{1}(\mathbb{R})\right) \text { s.t. } \hat{e} \in L^{\infty}\left(0, T ; H^{1}(\mathbb{R})\right) \text { and } e \in L^{\infty}\left(0, T ; H^{2}(\mathbb{R})\right)\right\} \text {. }
$$

The contraction property is obtained in $L^{\infty}\left(0, T ; L^{2}(\mathbb{R})\right)$.

If $H_{e 0} \in H_{n}^{2}(\Omega), E_{0} \in H^{1}(\mathbb{R})$ and $\hat{E}_{0} \in H^{2}(\Omega) \cap H^{1}(\mathbb{R})$, we also show that $\tau$ maps $B_{R}\left(V_{T}\right)$ into itself, for $R$ large enough and $T$ small enough, where $V_{T}$ is

$$
V_{T}=\left\{e \in L^{\infty}\left(0, T, H^{1}(\mathbb{R})\right) \text { s.t. } \hat{e} \in L^{\infty}\left(0, T, H^{1}(\mathbb{R}) \cap H^{2}(\Omega)\right)\right\} .
$$

\subsubsection{Uniform estimates in $\varepsilon$}

At this point, we have a solution to problem (3.4). We will obtain uniform estimates in $\varepsilon$ for the solutions of (3.4) in order to let $\varepsilon$ tends to 0 .

Proposition 3.3. The solution of (3.4) satisfies for all $t \leq T^{\star}$

$$
\begin{aligned}
& \left|E^{\varepsilon}(t)\right|_{H^{1}(\mathbb{R})}^{2}+\left|\hat{E}^{\varepsilon}(t)\right|_{H^{1}(\mathbb{R})}^{2}+\left|\partial_{\xi}^{2} \hat{E}^{\varepsilon}(t)\right|_{L^{2}(\Omega)}^{2}+\left|H_{e}(t)\right|_{L^{2}(\Omega)}^{2}+\left|\partial_{\xi}^{2} H_{e}(t)\right|_{L^{2}(\Omega)}^{2}+\int_{0}^{t} \int_{\Omega}\left(|\hat{E}|^{2}+\varepsilon\right)\left|\partial_{\xi}^{3} H_{e}\right|^{2} \\
& \quad \leq C(t)\left(\left|E_{0}\right|_{H^{1}(\mathbb{R})}^{2}+\left|\hat{E}_{0}\right|_{H^{1}(\mathbb{R})}^{2}+\left|\partial_{\xi}^{2} \hat{E}_{0}\right|_{L^{2}(\Omega)}^{2}+\left|H_{e 0}\right|_{L^{2}(\Omega)}^{2}+\left|\partial_{\xi}^{2} H_{e 0}\right|_{L^{2}(\Omega)}^{2}\right)+|S|_{H^{1}(\mathbb{R})}^{2}+|\hat{S}|_{H^{1}(\mathbb{R})}^{2}+\left|\partial_{\xi}^{2} \hat{S}\right|_{L^{2}(\Omega)}^{2}
\end{aligned}
$$

where the function $C(t)$ does not depend on $\varepsilon$.

The $L^{2}$ estimates on $\hat{E}^{\varepsilon}$ and $H_{e}^{\varepsilon}$ are

$$
\begin{gathered}
\frac{\mathrm{d}}{\mathrm{d} t}\left|\hat{E}^{\varepsilon}\right|_{L^{2}(\mathbb{R})}^{2} \leq 2\left|\hat{\nu}^{\varepsilon} \hat{E}^{\varepsilon}\right|_{L^{2}(\Omega)}\left|\hat{E}^{\varepsilon}\right|_{L^{2}(\Omega)}+|\hat{S}|_{L^{2}(\mathbb{R})}^{2}+\left|\hat{E}^{\varepsilon}\right|_{L^{2}(\mathbb{R})}^{2}, \\
\frac{\mathrm{d}}{\mathrm{d} t}\left|\xi^{-1} H_{e}^{\varepsilon}\right|_{L^{2}(\Omega)}^{2}+2 \int_{\Omega}\left(|\xi|^{3}\left|\hat{E}^{\varepsilon}\right|^{2}+\varepsilon\right)\left|\partial_{\xi} H_{e}^{\varepsilon}\right|^{2} \leq 0
\end{gathered}
$$

while the $H^{1}$ estimates give

$$
\begin{gathered}
\frac{\mathrm{d}}{\mathrm{d} t}\left|\partial_{x} E^{\varepsilon}\right|_{L^{2}(\mathbb{R})}^{2}+2 \int_{\Omega} \hat{\nu}_{e}^{\varepsilon} \hat{E}^{\varepsilon} \xi^{2} \overline{\hat{E}^{\varepsilon}} \leq 6 \int_{\mathbb{R}}\left|\partial_{x} E^{\varepsilon}\right|^{2}\left|E^{\varepsilon}\right|^{2}, \\
\frac{\mathrm{d}}{\mathrm{d} t}\left|\partial_{\xi} \hat{E}^{\varepsilon}\right|_{L^{2}(\mathbb{R})}^{2}+2 \int_{\Omega} \hat{\nu}^{\varepsilon}\left|\partial_{\xi} \hat{E}^{\varepsilon}\right|^{2}+2 \int_{\Omega} \partial_{\xi} \hat{\nu}^{\varepsilon} \hat{E}^{\varepsilon} \partial_{\xi} \overline{\hat{E}}=2 \int_{\mathbb{R}}\left(\partial_{\xi} \hat{E}^{\varepsilon} * \hat{E} * \overline{\hat{E}}\right) \partial_{\xi} \overline{\hat{E}} \\
\frac{\mathrm{d}}{\mathrm{d} t}\left|\partial_{\xi} H_{e}^{\varepsilon}\right|_{L^{2}(\Omega)}^{2}+2 \int_{\Omega} \xi^{2}\left(|\xi|^{3}|\hat{E}|^{2}+\varepsilon\right)\left|\partial_{\xi}^{2} H_{e}^{\varepsilon}\right|^{2} \leq \int \xi^{3}|\hat{E}|^{2}\left|\partial_{\xi} H_{e}^{\varepsilon}\right|^{2}+\left|\partial_{\xi}^{2} H_{e}^{\varepsilon}\right|_{L^{2}}^{2}\left|\partial_{\xi} \hat{E}^{\varepsilon}\right|_{L^{2}}^{2} .
\end{gathered}
$$


The different terms in (3.8) are controlled in the following way:

$$
-\int_{\Omega} \partial_{\xi} \hat{\nu}^{\varepsilon} \hat{E}^{\varepsilon} \partial_{\xi} \overline{\hat{E}^{\varepsilon}} \leq \int_{\Omega}|\xi|^{5}\left|\hat{E}^{\varepsilon}\right|^{2}\left|\partial_{\xi}^{2} \hat{H}_{e}^{\varepsilon}\right|^{2}+\left|\partial_{\xi} \hat{E}^{\varepsilon}\right|_{L^{2}(\mathbb{R})}^{2},
$$

since $\hat{\nu}^{\varepsilon}(\xi)=\operatorname{sgn}(\xi) \partial_{\xi} H_{e}^{\varepsilon}$, while the convolution is estimated as usual by

$$
\int_{\mathbb{R}}\left(\partial_{\xi} \hat{E}^{\varepsilon} * \hat{E} * \overline{\hat{E}^{\varepsilon}}\right) \partial_{\xi} \overline{\hat{E}^{\varepsilon}} \leq\left|\partial_{\xi} \hat{E}^{\varepsilon}\right|_{L^{2}(\mathbb{R})}^{2}\left|\hat{E}^{\varepsilon}\right|_{L^{1}(\mathbb{R})}^{2} \leq c\left|\partial_{\xi} \hat{E}^{\varepsilon}\right|_{L^{2}(\mathbb{R})}^{2}\left|E^{\varepsilon}\right|_{H^{1}(\mathbb{R})}^{2} .
$$

We remark that the estimate (3.10) is uniform in $\varepsilon$ thanks to the inequality (3.9).

Without an assumption on the sign of $\hat{\nu}_{e}^{\varepsilon}$, we get

$$
-\int_{\Omega} \hat{\nu}^{\varepsilon}\left|\partial_{\xi} \hat{E}^{\varepsilon}\right|^{2} \leq c\left|\hat{\nu}^{\varepsilon}\right|_{H^{1}(\Omega)}\left|\partial_{\xi} \hat{E}^{\varepsilon}\right|_{L^{2}(\mathbb{R})}^{2} .
$$

The latter term needs to establish a control in $H^{2}(\Omega)$ in order to obtain uniform estimates in $\varepsilon$ of $\hat{\nu}^{\varepsilon}$. We obtain

$$
\begin{aligned}
\frac{\mathrm{d}}{\mathrm{d} t}\left|\partial_{\xi}^{2} \hat{E}^{\varepsilon}\right|_{L^{2}(\Omega)}^{2}+2 \int_{\Omega} \hat{\nu}^{\varepsilon}\left|\partial_{\xi}^{2} \hat{E}^{\varepsilon}\right|^{2}+4 \int_{\Omega} \partial_{\xi} \hat{\nu}^{\varepsilon} \partial_{\xi} \hat{E}^{\varepsilon} \partial_{\xi}^{2} \overline{\hat{E}^{\varepsilon}} & +2 \int_{\Omega} \partial_{\xi}^{2} \hat{\nu}^{\varepsilon} \hat{E}^{\varepsilon} \partial_{\xi}^{2} \overline{\hat{E}^{\varepsilon}} \\
& =2 \int_{\Omega}\left(\partial_{\xi} \hat{E}^{\varepsilon} * \partial_{\xi} \hat{E}^{\varepsilon} * \overline{\hat{E}^{\varepsilon}}\right) \partial_{\xi}^{2} \overline{\hat{E}^{\varepsilon}}+2 \int_{\Omega} \partial_{\xi}^{2} \hat{S} \partial_{\xi}^{2} \overline{\hat{E}^{\varepsilon}}
\end{aligned}
$$

and

$$
\begin{aligned}
& \frac{\mathrm{d}}{\mathrm{d} t}\left|\partial_{\xi}^{2} H_{e}^{\varepsilon}\right|_{L^{2}(\Omega)}^{2}+2 \int_{\Omega} \xi^{2}\left(|\xi|^{3}|\hat{E}|^{2}+\varepsilon\right)\left|\partial_{\xi}^{3} H_{e}^{\varepsilon}\right|^{2}+\left|\partial_{\xi}^{2} H_{e}^{\varepsilon}\right|_{L^{2}(\Omega)}^{2} \\
& \quad \leq 4 \int_{\Omega}\left|\left(1+\partial_{\xi}\right)\left(|\xi|^{3}|\hat{E}|^{2}+\varepsilon\right)\right|\left|\partial_{\xi}^{2} H_{e}^{\varepsilon}\right|\left|\partial_{\xi}^{3} H_{e}^{\varepsilon}\right|+4 \int_{\Omega}\left|\partial_{\xi}\left(|\xi|^{3}|\hat{E}|^{2}+\varepsilon\right)\right|\left|\partial_{\xi} H_{e}^{\varepsilon}\right|\left|\partial_{\xi}^{3} H_{e}^{\varepsilon}\right|+\left|\partial_{\xi}^{2} H_{e 0}\right|_{L^{2}(\Omega)}^{2}
\end{aligned}
$$

The control of the various terms of (3.12) is done in the following way:

$$
\int_{\Omega} \hat{\nu}^{\varepsilon}\left|\partial_{\xi}^{2} \hat{E}^{\varepsilon}\right|^{2} \leq c\left|\partial_{\xi} H_{e}^{\varepsilon}\right|_{H^{1}(\Omega)}\left|\partial_{\xi}^{2} \hat{E}^{\varepsilon}\right|_{L^{2}(\Omega)}^{2}
$$

since $\hat{\nu}^{\varepsilon}(\xi)=\operatorname{sgn}(\xi) \partial_{\xi} H_{e}^{\varepsilon}$. Moreover

$$
\int_{\Omega} \partial_{\xi} \hat{\nu}^{\varepsilon} \partial_{\xi} \hat{E}^{\varepsilon} \partial_{\xi}^{2} \overline{\hat{E}^{\varepsilon}} \leq\left|\partial_{\xi}^{2} H_{e}^{\varepsilon}\right|_{L^{2}(\Omega)}^{2}+c\left|\partial_{\xi} \hat{E}^{\varepsilon}\right|_{H^{1}(\Omega)}^{2}\left|\partial_{\xi}^{2} \hat{E}^{\varepsilon}\right|_{L^{2}(\Omega)}^{2},
$$

and

$$
\int_{\Omega} \partial_{\xi}^{2} \hat{\nu}^{\varepsilon} \hat{E}^{\varepsilon} \partial_{\xi}^{2} \overline{\hat{E}^{\varepsilon}} \leq \int_{\Omega}|\xi|^{5}\left|\hat{E}^{\varepsilon}\right|^{2}\left|\partial_{\xi}^{3} H_{e}^{\varepsilon}\right|^{2}+\left|\partial_{\xi}^{2} \hat{E}^{\varepsilon}\right|_{L^{2}(\Omega)}^{2} .
$$

In order to control the last term of (3.12), we use the fact that the domain $\Omega$ is bounded:

$$
\begin{aligned}
\int_{\Omega}\left(\partial_{\xi} \hat{E}^{\varepsilon} * \partial_{\xi} \hat{E}^{\varepsilon} * \overline{\hat{E}^{\varepsilon}}\right) \partial_{\xi}^{2} \overline{\hat{E}^{\varepsilon}} & \leq\left.\left|\partial_{\xi} \hat{E}^{\varepsilon} * \partial_{\xi} \hat{E}^{\varepsilon} * \overline{\hat{E}^{\varepsilon}}\right|_{L^{2}(\Omega)}|| \partial_{\xi}^{2} \hat{E}^{\varepsilon}\right|_{L^{2}(\Omega)} \\
& \leq\left.\left.|\Omega|^{\frac{1}{2}}|| \partial_{\xi} \hat{E}^{\varepsilon} * \partial_{\xi} \hat{E}^{\varepsilon} * \overline{\hat{E}^{\varepsilon}}\right|_{L^{\infty}(\mathbb{R})}|| \partial_{\xi}^{2} \hat{E}^{\varepsilon}\right|_{L^{2}(\Omega)} \\
& \leq\left.|\Omega|^{\frac{1}{2}}|| \partial_{\xi} \hat{E}^{\varepsilon}\right|_{L^{2}(\mathbb{R})} ^{2}\left|\hat{E}^{\varepsilon}\right|_{L^{1}(\mathbb{R})}\left|\partial_{\xi}^{2} \hat{E}^{\varepsilon}\right|_{L^{2}(\Omega)}
\end{aligned}
$$


Moreover,

$$
\left|\hat{E}^{\varepsilon}\right|_{L^{1}(\mathbb{R})} \leq c\left|E^{\varepsilon}\right|_{H^{1}(\mathbb{R})}
$$

This finishes the control of the terms of (3.12).

Using (3.13) it follows that,

$$
\begin{aligned}
\int_{\Omega} \mid(1+ & \left.\partial_{\xi}\right)\left(|\xi|^{3}|\hat{E}|^{2}\right)|| \partial_{\xi}^{2} H_{e}^{\varepsilon}|| \partial_{\xi}^{3} H_{e}^{\varepsilon}\left|+\int_{\Omega}\right| \partial_{\xi}\left(|\xi|^{3}|\hat{E}|^{2}\right)|| \partial_{\xi} H_{e}^{\varepsilon}|| \partial_{\xi}^{3} H_{e}^{\varepsilon} \mid \\
& \leq 8 \int_{\Omega}\left(\left|\hat{E^{\varepsilon}}\right|+\left|\partial \hat{E}^{\varepsilon}\right|\right)\left(\left|\partial_{\xi} H_{e}^{\varepsilon}\right|+\left|\partial_{\xi}^{2} H_{e}^{\varepsilon}\right|\right)\left(\left|\hat{E}^{\varepsilon}\right|\left|\partial_{\xi}^{3} H_{e}^{\varepsilon}\right|\right) \\
& \leq c\left|\hat{E^{\varepsilon}}\right|_{H^{2}(\Omega)}^{4}+\left|H_{e}^{\varepsilon}\right|_{H^{2}(\Omega)}^{4}+\frac{1}{4} \int_{\Omega}|\xi|^{5}\left|\hat{E}^{\varepsilon}\right|^{2}\left|\partial_{\xi}^{3} H_{e}^{\varepsilon}\right|^{2}
\end{aligned}
$$

Thus, summing the various estimates we have obtained, we get

$$
\begin{gathered}
\frac{\mathrm{d}}{\mathrm{d} t}\left(\left|E^{\varepsilon}\right|_{H^{1}(\mathbb{R})}^{2}+\left|\hat{E^{\varepsilon}}\right|_{H^{1}(\mathbb{R})}^{2}+\left|\partial_{\xi}^{2} \hat{E^{\varepsilon}}\right|_{L^{2}(\Omega)^{2}}+\left|\xi^{-1} H_{e}^{\varepsilon}\right|_{L^{2}(\Omega)}^{2}+\left|\partial_{\xi} H_{e}^{\varepsilon}\right|_{H^{1}(\Omega)}^{2}\right) \\
+\int_{\Omega}\left(\left|\hat{E}^{\varepsilon}\right|^{2}+\varepsilon\right)\left(\left|\partial_{\xi}^{3} H_{e}^{\varepsilon}\right|^{2}+\left|\partial_{\xi}^{2} H_{e}^{\varepsilon}\right|^{2}+\left|\partial_{\xi} H_{e}^{\varepsilon}\right|^{2}\right) \\
\leq c\left(\left|\hat{E^{\varepsilon}}\right|_{H^{1}(\mathbb{R})}^{4}+\left|\partial_{\xi}^{2} \hat{E^{\varepsilon}}\right|_{L^{2}(\Omega)}^{4}+\left|H_{e}^{\varepsilon}\right|_{H^{2}(\Omega)}^{4}+\left|E^{\varepsilon}\right|_{H^{1}(\mathbb{R})}^{4}+|S|_{H^{1}(\mathbb{R})}^{2}+|\hat{S}|_{H^{1}(\mathbb{R})}^{2}+\left|\partial_{\xi}^{2} \hat{S}\right|_{L^{2}(\Omega)}^{2}\right) .
\end{gathered}
$$

One easily deduces the estimate (3.6).

Passing to the limit $\varepsilon$ to 0 , we obtain the local existence result of Theorem 1.1.

We still have to prove the uniqueness result.

Let $\left(E_{1}, \hat{E}_{1}, H_{e 1}, \nu_{1}\right)$ and $\left(E_{2}, \hat{E}_{2}, H_{e 2}, \nu_{2}\right)$ be two solutions of $(3.1)$ and denote by $\left(E, \hat{E}, H_{e}, \nu\right)$ the difference of these solutions. Then $\left(E, \hat{E}, H_{e}, \nu\right)$ satisfies:

$$
\begin{aligned}
& i\left(\partial_{t} E+\nu_{1} * E+\nu * E_{2}\right)+\partial_{x}^{2} E=-\left|E_{1}\right|^{2} E-\left(E_{1} \bar{E}+E \overline{E_{2}}\right) E_{2}, \\
& \partial_{t} H_{e}-\frac{1}{2} \xi^{2} \partial_{\xi}\left(|\xi|^{3}\left|\hat{E}_{2}\right|^{2} \partial_{\xi} H_{e}+|\xi|^{3}\left(\left|\hat{E}_{1}\right|^{2}-\left|\hat{E}_{2}\right|^{2}\right) \partial_{\xi} H_{e 1}\right) \\
& -\frac{1}{2} \xi^{2} \partial_{\xi}\left(|\xi|^{3}\left|\hat{E}_{1}\right|^{2} \partial_{\xi} H_{e}+|\xi|^{3}\left(\left|\hat{E}_{2}\right|^{2}-\left|\hat{E}_{1}\right|^{2}\right) \partial_{\xi} H_{e 2}\right)=0, \quad \forall \xi \in \Omega, \\
& \partial_{\xi} H_{e} \mid \partial \Omega=0, \\
& H_{e}(0, .)=0, E(0, .)=0 .
\end{aligned}
$$

Performing an $L^{2}$ estimate of $\left(E, \hat{E}, H_{e}, \nu\right)$ yields

$$
\begin{gathered}
\frac{\mathrm{d}}{\mathrm{d} t}|\hat{E}|_{L^{2}(\mathbb{R})}^{2} \leq 2 \int_{\Omega}\left|\hat{\nu}_{1}\right||\hat{E}|^{2}+\left.|\hat{\nu}||| \hat{E}_{2}|| \hat{E}|+| E_{1}\right|_{L^{\infty}(\mathbb{R})}|\hat{E}|_{L^{2}(\mathbb{R})}^{2} \leq C|\hat{E}|_{L^{2}(\mathbb{R})}^{2}+\int_{\Omega}\left|\hat{E}_{2}\right|^{2}|\hat{\nu}|^{2}, \\
\frac{\mathrm{d}}{\mathrm{d} t}\left|\xi^{-1} H_{e}\right|_{L^{2}(\mathbb{R})}^{2}+\int_{\Omega} \xi^{3}\left(\left|\hat{E}_{1}\right|^{2}+\left|\hat{E}_{2}\right|^{2}\right)\left|\partial_{\xi} H_{e}\right|^{2}+2\left|H_{e}\right|_{L^{2}(\Omega)}^{2} \\
\leq\left.\int_{\Omega} \xi^{3}|| \hat{E}_{1}\right|^{2}-\left|\hat{E}_{2}\right|^{2}\left|\left(\left|\partial_{\xi} H_{e 1}\right|+\left|\partial_{\xi} H_{e 2}\right|\right)\right| \partial_{\xi} H_{e} \mid \\
\leq C|\hat{E}|_{L^{2}(\Omega)}^{2}+\frac{1}{2} \int_{\Omega} \xi^{3}\left(\left|\hat{E}_{1}\right|^{2}+\left|\hat{E}_{2}\right|^{2}\right)\left|\partial_{\xi} H_{e}\right|^{2} .
\end{gathered}
$$

Thus, by making the sum of these estimates, one gets

$$
\frac{\mathrm{d}}{\mathrm{d} t}\left(|\hat{E}|_{L^{2}(\mathbb{R})}^{2}+\left|\xi^{-1} H_{e}\right|_{L^{2}(\mathbb{R})}^{2}\right)+\int_{\Omega} \xi^{3}\left|\hat{E}_{2}\right|^{2}\left|\partial_{\xi} H_{e}\right|^{2} \leq C|\hat{E}|_{L^{2}(\Omega)}^{2},
$$


where $C$ depends on the solutions $\left(E_{i}, \hat{E}_{i}, H_{e i}\right)(i=1,2)$, in the norm of $H^{1}(\mathbb{R}) \times H^{2}(\Omega) \cap H^{1}(\mathbb{R}) \times H^{2}(\Omega)$.

The uniqueness result is then straightforward.

\subsection{Global existence of strong solutions for the homogeneous problem}

The aim of this section is to prove Theorem 1.2. We just perform a priori estimates (these estimates can be done on the approximate solutions constructed in the preceding section for example).

- Step 1. Maximum principle.

Proposition 3.4. If $H$ is a solution to

$$
\partial_{t} H-\xi^{2} \partial_{\xi}\left(|\xi|^{3}|\hat{E}|^{2} \partial_{\xi} H\right)=0, \quad \forall \xi \in \Omega,
$$

and $\operatorname{sgn}(\xi) \partial_{\xi} H(0, \xi) \geq 0$, then for all $t \geq 0, \xi \in \Omega$

$$
\hat{\nu}(t, \xi)=\operatorname{sgn}(\xi) \partial_{\xi} H(t, \xi) \geq 0 .
$$

Proof. The equation satisfied by $\widehat{\nu}$ is

$$
\partial_{t} \widehat{\nu}-\xi^{2} \partial_{\xi}\left(|\xi|^{3}|\widehat{E}|^{2} \partial_{\xi} \widehat{\nu}\right)=\xi^{2} \partial_{\xi}\left(\left(\partial_{\xi}\left(\xi^{3}|\widehat{E}|^{2}\right) \widehat{\nu}\right)\right)+2 \xi \partial_{\xi}\left(\xi^{3}|\widehat{E}|^{2} \widehat{\nu}\right) .
$$

By substituting $\widehat{\nu}$ by $\widehat{\nu}=\widehat{\nu}^{+}-\widehat{\nu}^{-}$, where $\widehat{\nu}^{+}, \widehat{\nu}^{-}$are the positive part and negative part of $\widehat{\nu}$ respectively, multiplying the equation by $-\xi^{-2}{\widehat{\nu_{e}}}^{-}$and integrating on $\Omega$ yields

$$
\begin{aligned}
\frac{1}{2} \frac{\mathrm{d}}{\mathrm{d} t}\left|\xi^{-1} \widehat{\nu}^{-}\right|_{L^{2}}^{2}+\int \xi^{3}|\widehat{E}|^{2}\left|\partial_{\xi} \widehat{\nu}^{-}\right|^{2} \mathrm{~d} \xi= & \int \widehat{\nu}^{-} \partial_{\xi} \widehat{\nu}^{-} \partial_{\xi}\left(\xi^{3}|\widehat{E}|^{2}\right) \mathrm{d} \xi \\
& +2 \int \widehat{\nu}^{-} \partial_{\xi}\left(\xi^{-1} \widehat{\nu}^{-}\right)\left(\xi^{3}|\widehat{E}|^{2}\right) \mathrm{d} \xi .
\end{aligned}
$$

This gives

$$
\frac{1}{2} \frac{\mathrm{d}}{\mathrm{d} t}\left|\xi^{-1} \widehat{\nu}^{-}\right|_{L^{2}}^{2}+\int \xi^{3}|\widehat{E}|^{2}\left|\partial_{\xi} \widehat{\nu}^{-}\right|^{2} \mathrm{~d} \xi \leq C(\Omega) \max \left(|\widehat{E}|_{L^{\infty}},\left|\partial_{\xi} \widehat{E}\right|_{L^{\infty}}\right)\left|\widehat{\nu}^{-}\right|_{L^{2}}^{2}
$$

Then it follows

$$
\frac{\mathrm{d}}{\mathrm{d} t}\left|\widehat{\nu}^{-}\right|_{L^{2}}^{2} \leq \alpha(\Omega)\left|\widehat{\nu}^{-}\right|_{L^{2}}^{2}
$$

According to Gronwall's lemma with $\widehat{\nu}^{-}(., 0)=0$, one gets the result.

- Step 2. We establish a $L_{l o c}^{\infty}\left(\mathbb{R}^{+}, H^{1}\right)$ bound for $\hat{E}$.

If $E$ satisfies

taking the Fourier transform yields

$$
i\left(\partial_{t} E+\nu * E\right)+\partial_{x}^{2} E=0,
$$

$$
i\left(\partial_{t} \hat{E}+\widehat{\nu} \hat{E}\right)-\xi^{2} \hat{E}=0
$$

Since $\widehat{\nu} \geq 0$, we have

$$
|\hat{E}|_{L^{2}}(t) \leq|\hat{E}|_{L^{2}}(0)
$$

Applying $\partial_{\xi}$ on (3.15), multiplying by $\partial_{\xi} \overline{\hat{E}}$ and taking the imaginary part yields

$$
\frac{1}{2} \frac{\mathrm{d}}{\mathrm{d} t} \int\left|\partial_{\xi} \hat{E}\right|^{2}+\int \widehat{\nu}\left|\partial_{\xi} \hat{E}\right|^{2}+\Re \int \partial_{\xi} \hat{\nu} \hat{E} \partial_{\xi} \overline{\hat{E}}=0,
$$


which implies

$$
\frac{1}{2} \frac{\mathrm{d}}{\mathrm{d} t} \int\left|\partial_{\xi} \hat{E}\right|^{2} \leq \alpha \int\left|\partial_{\xi} \widehat{\nu}\right|^{2}|\hat{E}|^{2}+\frac{1}{\alpha}\left|\partial_{\xi} \hat{E}\right|^{2}
$$

since $\int \widehat{\nu}\left|\partial_{\xi} \hat{E}\right|^{2} \geq 0$ and $\alpha$ will be fixed latter one.

Remark that the term $\int\left|\partial_{\xi} \widehat{\nu}\right|^{2}|\hat{E}|^{2}$ will be controlled thanks to the dissipative term on the equation on $H$.

- Step 3. $L_{l o c}^{\infty}\left(\mathbb{R}^{+}, L^{2}\right)$ estimate for $H$.

Multiplying (1.14) by $\frac{H}{\xi^{2}}$ and integrating on $\Omega$ gives

$$
\frac{1}{2} \frac{\mathrm{d}}{\mathrm{d} t} \int \frac{H^{2}}{\xi^{2}}+\int \xi^{3}|\hat{E}|^{2}\left|\partial_{\xi} H\right|^{2}=0
$$

- Step 4. $L_{\text {loc }}^{\infty}\left(\mathbb{R}^{+}, H^{1}\right)$ estimate for $H$.

We apply $\partial_{\xi}$ on (1.14) and multiply the result by $\partial_{\xi} H$. This leads to

$$
\frac{1}{2} \frac{\mathrm{d}}{\mathrm{d} t} \int\left|\partial_{\xi} H\right|^{2}-\int \partial_{\xi}\left(\xi^{2} \partial_{\xi}\left(\xi^{3}|\hat{E}|^{2} \partial_{\xi} H\right)\right) \partial_{\xi} H=0
$$

that is

or

$$
\begin{gathered}
\frac{1}{2} \frac{\mathrm{d}}{\mathrm{d} t} \int\left|\partial_{\xi} H\right|^{2}+\int \xi^{2} \partial_{\xi}\left(\xi^{3}|\hat{E}|^{2} \partial_{\xi} H\right) \partial_{\xi}^{2} H=0 \\
\frac{1}{2} \frac{\mathrm{d}}{\mathrm{d} t} \int\left|\partial_{\xi} H\right|^{2}+\int \xi^{5}|\hat{E}|^{2}\left|\partial_{\xi}^{2} H\right|^{2}+\int \xi^{2} \partial_{\xi}\left(\xi^{3}|\hat{E}|^{2}\right) \partial_{\xi} H \partial_{\xi}^{2} H=0 .
\end{gathered}
$$

The crucial point is to deal with the term $\int \xi^{2} \partial_{\xi}\left(\xi^{3}|\hat{E}|^{2}\right) \partial_{\xi} H \partial_{\xi}^{2} H$. We strongly use the structure of the system (1.13)-(1.14).

Taking the Fourier transform of (1.13), multiplying by $\overline{\hat{E}}$ and taking the imaginary part gives

$$
\frac{1}{2} \frac{\mathrm{d}}{\mathrm{d} t}|\hat{E}|^{2}+\partial_{\xi} H|\hat{E}|^{2}=0
$$

We consider $\xi^{2} \partial_{\xi}\left(\xi^{3}(3.17)\right)$ and obtain

$$
\frac{1}{2} \frac{\mathrm{d}}{\mathrm{d} t}\left(\xi^{2} \partial_{\xi}\left(\xi^{3}\left(|\hat{E}|^{2}\right)\right)\right)+\xi^{2} \partial_{\xi}\left(\xi^{3} \partial_{\xi} H|\hat{E}|^{2}\right)=0
$$

and using (1.14), one gets

It follows that

$$
\frac{1}{2} \frac{\mathrm{d}}{\mathrm{d} t}\left(\xi^{2} \partial_{\xi}\left(\xi^{3}\left(|\hat{E}|^{2}\right)\right)\right)+\partial_{t} H=0
$$

$$
\xi^{2} \partial_{\xi}\left(\xi^{3}\left(|\hat{E}|^{2}\right)\right)+2 H=f_{0},
$$

where $f_{0}=\xi^{2} \partial_{\xi}\left(\xi^{3}\left(\left|\hat{E}_{0}\right|^{2}\right)\right)+2 H_{0}$.

Plugging (3.22) in (3.18) leads to

$$
\frac{1}{2} \frac{\mathrm{d}}{\mathrm{d} t} \int\left|\partial_{\xi} H\right|^{2}+\int \xi^{5}|\hat{E}|^{2}\left|\partial_{\xi}^{2} H\right|^{2}-2 \int H \partial_{\xi} H \partial_{\xi}^{2} H+\int f_{0} \partial_{\xi} H \partial_{\xi}^{2} H=0 .
$$

Integration by parts gives

$$
\frac{1}{2} \frac{\mathrm{d}}{\mathrm{d} t} \int\left|\partial_{\xi} H\right|^{2}+\int \xi^{5}|\hat{E}|^{2}\left|\partial_{\xi}^{2} H\right|^{2}+\int\left(\partial_{\xi} H\right)^{3}-\frac{1}{2} \int \partial_{\xi} f_{0}\left|\partial_{\xi} H\right|^{2}=0 .
$$


Recall now that thanks to Step $1, \partial_{\xi} H \geq 0$, and one gets

$$
\frac{1}{2} \frac{\mathrm{d}}{\mathrm{d} t} \int\left|\partial_{\xi} H\right|^{2}+\int \xi^{5}|\hat{E}|^{2}\left|\partial_{\xi}^{2} H\right|^{2} \leq \frac{1}{2} \int\left(\partial_{\xi} f_{0}\right)^{+}\left|\partial_{\xi} H\right|^{2} .
$$

Inequalities (3.16)-(3.24) give

$$
\frac{\mathrm{d}}{\mathrm{d} t}\left(|\hat{E}|^{2}+\left|\partial_{\xi} H\right|^{2}\right)+\int \xi^{5}|\hat{E}|^{2}\left|\partial_{\xi}^{2} H\right|^{2} \leq \alpha \int\left|\partial_{\xi} \widehat{\nu}\right|^{2}|\hat{E}|^{2}+\frac{1}{\alpha}\left|\partial_{\xi} \hat{E}\right|^{2}+\frac{1}{2} \int\left(\partial_{\xi} f_{0}\right)^{+}\left|\partial_{\xi} H\right|^{2} .
$$

Since $\xi \in[1,2]$, we can choose $\alpha=1$. One gets bounds for $\hat{E}, H$ in $L_{l o c}^{\infty}\left(\mathbb{R}^{+}, H^{1}\right)$ and the result follows.

\section{NUMERICAL APPROXIMATION}

The discretization of the Zakharov equations

$$
\begin{gathered}
\left(i \partial_{t}+\partial_{x}^{2}\right) E=\delta n E \\
\left(\partial_{t}^{2}-\mu \partial_{x}^{2}\right) \delta n=\mu \partial_{x}^{2}|E|^{2}
\end{gathered}
$$

is now well understood. Our main contribution is here the coupling with the diffusion model. The discretization of the Zakharov system that we use is that of Glassey [9]. In this section, using a time-splitting spectral discretization for the Zakharov system and a finite difference scheme for the electron diffusion equation, we present numerical simulations and we show how Landau damping works.

We consider the full system with periodic boundary conditions:

$$
\begin{aligned}
& i\left(\partial_{t} E+\nu * E\right)+\partial_{x}^{2} E=\delta n E+E_{p}(x) \mathrm{e}^{i\left(k_{1} x-\omega_{1} t\right)}, 0<x<L, t>0, \\
& \partial_{t}^{2} \delta n-\mu \partial_{x}^{2} \delta n=\mu \partial_{x}^{2}\left(|E|^{2}\right), 0<x<L, t>0, \\
& \widehat{\nu}(t, \xi)=\operatorname{sgn}(\xi) \partial_{\xi} H_{e}(t, \xi), \xi \in \Omega, t>0, \\
& \partial_{t} H_{e}-\xi^{2} \partial_{\xi}\left(|\xi|^{3}|\hat{E}|^{2} \partial_{\xi} H_{e}\right)=0, \xi \in \Omega, \\
& E(t, 0)=E(t, L), t>0, \\
& \delta n(t, 0)=\delta n(t, L), t>0 .
\end{aligned}
$$

System (4.1)-(4.4) is endowed with the following initial conditions:

$$
E(0, x)=E_{0}(x), \delta n(0, x)=\delta n_{0}(x), \partial_{t} \delta n(0, x)=\delta n_{1}(x), H_{e}(0, \xi)=H_{e, 0}(\xi) .
$$

Moreover we suppose that

$$
E_{0}(0)=E_{0}(L), \delta n_{0}(0)=\delta n_{0}(L), \delta n_{1}(0)=\delta n_{1}(L)
$$

It is easy to show that the above system satisfies the following 
Proposition 4.1. If $\int_{0}^{L} \delta n_{1}(x) \mathrm{d} x=0$, any regular solution of (4.1)-(4.6) satisfies:

$$
\begin{aligned}
& \int_{0}^{L} \delta n(t, x) \mathrm{d} x=\int_{0}^{L} \delta n_{0}(x) \mathrm{d} x, \forall t \geq 0, \\
& \int \frac{1}{\xi^{2}} H_{e}(t, \xi) \mathrm{d} \xi=\int \frac{1}{\xi^{2}} H_{e}(0, \xi) \mathrm{d} \xi, \forall t \geq 0 .
\end{aligned}
$$

Moreover if $E_{p}=0$, then

$$
\frac{1}{2} \frac{\mathrm{d}}{\mathrm{d} t} \int \frac{1}{\xi^{4}} H_{e}(t, \xi) \mathrm{d} \xi=\int \widehat{\nu}(t, \xi)|\hat{E}|^{2} \mathrm{~d} \xi, \forall t \geq 0
$$

Below, we construct a numerical approximation of (4.1)-(4.4) which is consistent with the above properties.

We choose the spatial mesh size $h=\triangle x$ with $h=L / N$ for $N=2 M$ being an even number, the time step being $\Delta t>0$ and let the grid points and the time step be

$$
x_{j}=j h, t_{k}=k \Delta t, j=1, \ldots, N, k=0,1,2, \ldots
$$

We use the sequence $\left(\xi_{j}=\frac{2 \pi j}{L}\right)_{j=-\frac{N}{2}, \ldots, 0, \ldots, \frac{N}{2}-1}$ as the regular mesh grid in frequency with $\triangle \xi=\frac{2 \pi}{L}$. Furthermore, let $E_{j}^{k}, \delta n_{j}^{k}$, and $\widehat{\nu}_{j}^{k}$ be the approximations of $E\left(t_{k}, x_{j}\right), \delta n\left(t_{k}, x_{j}\right), \widehat{\nu}\left(t_{k}, \xi_{j}\right)$. Then, in order to be consistent with the evaluation of $\widehat{\nu}\left(t_{k}, \xi_{j}\right)=\operatorname{sgn}\left(\xi_{j}\right) \frac{\pi}{2} \partial_{\xi} H\left(t_{k}, \xi_{j}\right)$, we approximate $H_{e}$ on the grid $\left(\xi_{j+\frac{1}{2}}\right)_{j}$ defined by $\xi_{j+\frac{1}{2}}=\frac{2 \pi(j+1 / 2)}{L}$.

\subsection{Finite difference scheme for the diffusion equation}

In order to evaluate the approximation of $H\left(t_{k}, \xi_{j+\frac{1}{2}}\right)$, we use an implicit difference scheme for the diffusion equation:

$$
\partial_{t} H_{e}-\xi^{2} \partial_{\xi}\left(|\xi|^{3}|\widehat{E}|^{2} \partial_{\xi} H_{e}\right)=0, \quad \xi \in \Omega
$$

where $\Omega=\left[-\xi_{2},-\xi_{1}\right] \cup\left[\xi_{1}, \xi_{2}\right],\left(\xi_{2}>\xi_{1}>0\right)$, with $\xi_{1}=\frac{2 \pi\left(j_{1}+1 / 2\right)}{L}>\frac{2 \pi}{L}, \xi_{2}=\frac{2 \pi\left(j_{2}+1 / 2\right)}{L}<\frac{2 \pi(M-1)}{L}$.

The initial electron distribution function is assumed to be a Maxwellian distribution:

$$
H_{e, 0}(\xi)=\frac{1}{\sqrt{2 \pi}} \exp \left(-\frac{1}{2 \xi^{2}}\right) \text {. }
$$

The boundary conditions are

$$
\begin{aligned}
& \partial_{\xi} H_{e}\left(., \pm \xi_{1}\right)=0, \\
& H_{e}\left(., \xi_{2}\right)=H_{e}\left(.,-\xi_{2}\right)
\end{aligned}
$$

The scheme reads:

$$
\frac{1}{\triangle t}\left(H_{j+\frac{1}{2}}^{k+1}-H_{j+\frac{1}{2}}^{k}\right)+(A H)_{j+\frac{1}{2}}^{k+1}=0
$$

where $(A H)_{j+\frac{1}{2}}^{k+1}$ is a discretization of $-\xi^{2} \partial_{\xi}\left(\beta(t, \xi) \partial_{\xi} H_{e}\right)$ in a conservative form at the point $\xi_{j+\frac{1}{2}}$ and time $t_{k+1}$. Here $\beta(t, \xi)=|\xi|^{3}|\widehat{E}|^{2}$. We choose $A$ such that:

$$
(A H)_{j+\frac{1}{2}}^{k}=-\frac{\xi_{j+\frac{1}{2}}^{2}}{\xi_{j+1}-\xi_{j}}\left[\beta_{j+1}^{k-1} \frac{H_{j+\frac{3}{2}}^{k}-H_{j+\frac{1}{2}}^{k}}{\xi_{j+\frac{3}{2}}-\xi_{j+\frac{1}{2}}}-\beta_{j}^{k-1} \frac{H_{j+\frac{1}{2}}^{k}-H_{j-\frac{1}{2}}^{k}}{\xi_{j+\frac{1}{2}}-\xi_{j-\frac{1}{2}}}\right],
$$


where $\beta_{j}^{k}$ is the discretization of $\beta(t, \xi)$ given in Proposition 4.2. Then we can evaluate $\widehat{\nu}\left(\xi_{j}, t^{k+1}\right)$ with the centered finite difference scheme:

$$
\widehat{\nu}_{j}^{k+1}=\operatorname{sgn}\left(\xi_{j}\right) \frac{H_{j+\frac{1}{2}}^{k+1}-H_{j-\frac{1}{2}}^{k+1}}{\triangle \xi}
$$

The next proposition shows that there exists some approximate values of $\beta(t, \xi)$ such that one obtains discrete equivalent of (4.8)-(4.9).

Proposition 4.2. (energy and density conservation). Take

$$
\beta_{l}^{k}=\frac{\xi_{l+\frac{1}{2}}^{2} \xi_{l-\frac{1}{2}}^{2}}{\left|\xi_{l+\frac{1}{2}}+\xi_{l-\frac{1}{2}}\right|}\left|\hat{E}_{l}^{k}\right|^{2}
$$

Then the following identities hold

$$
\begin{gathered}
\sum_{l} \frac{1}{\xi_{l+\frac{1}{2}}^{2}} H_{l+\frac{1}{2}}^{k+1}=\sum_{l} \frac{1}{\xi_{l+\frac{1}{2}}^{2}} H_{l+\frac{1}{2}}^{k}, \\
\sum_{l} \frac{1}{\xi_{l+\frac{1}{2}}^{4}} H_{l+\frac{1}{2}}^{k+1}=\sum_{l} \frac{1}{\xi_{l+\frac{1}{2}}^{4}} H_{l+\frac{1}{2}}^{k}+\Delta t \sum_{l} \widehat{\nu}_{l}^{k+1}\left|\hat{E}_{l}^{k}\right|^{2} .
\end{gathered}
$$

Proof. The first identity follows from the conservativity of the discretization of $\partial_{\xi}\left(\beta(\xi, t) \partial_{\xi} H_{e}\right)$ given by (4.11). Below, we will prove the second conservation law.

According to the scheme (4.10)-(4.12), we have

$$
\frac{H_{j+\frac{1}{2}}^{k+1}}{\xi_{j+\frac{1}{2}}^{4}}=\frac{H_{j+\frac{1}{2}}^{k}}{\xi_{j+\frac{1}{2}}^{4}}+\triangle t\left(\frac{\beta_{j+1}^{k}}{\xi_{j+\frac{1}{2}}^{2}} \frac{H_{j+\frac{3}{2}}^{k+1}-H_{j+\frac{1}{2}}^{k+1}}{\triangle \xi^{2}}-\frac{\beta_{j}^{k}}{\xi_{j+\frac{1}{2}}^{2}} \frac{H_{j+\frac{1}{2}}^{k+1}-H_{j-\frac{1}{2}}^{k+1}}{\triangle \xi^{2}}\right) .
$$

Taking into account boundary conditions, it follows that

$$
\sum_{l} \frac{H_{l+\frac{1}{2}}^{k+1}}{\xi_{l+\frac{1}{2}}^{4}}=\sum_{l} \frac{H_{l+\frac{1}{2}}^{k}}{\xi_{l+\frac{1}{2}}^{4}}+\triangle t\left(\sum_{l} \beta_{l}^{k}\left(\frac{1}{\xi_{l-\frac{1}{2}}^{2}}-\frac{1}{\xi_{l+\frac{1}{2}}^{2}}\right) \frac{H_{l+\frac{1}{2}}^{k+1}-H_{l-\frac{1}{2}}^{k+1}}{\triangle \xi^{2}}\right) .
$$

Plugging (4.12) in (4.15) yields

$$
\sum_{l} \frac{H_{l+\frac{1}{2}}^{k+1}}{\xi_{l+\frac{1}{2}}^{4}}=\sum_{l} \frac{H_{l+\frac{1}{2}}^{k}}{\xi_{l+\frac{1}{2}}^{4}}+\Delta t\left(\sum_{l} \beta_{l}^{k} \operatorname{sgn}\left(\xi_{l}\right)\left(\frac{\xi_{l+\frac{1}{2}}^{2} \xi_{l-\frac{1}{2}}^{2}}{\xi_{l+\frac{1}{2}}+\xi_{l-\frac{1}{2}}}\right)^{-1} \widehat{\nu}_{l}^{k+1}\right)
$$

and with the choice of discretization of $\beta(t, \xi)$, the result follows.

Proposition 4.3. (maximum principle). Let $\widehat{\nu}_{0}=\widehat{\nu}(t=0)$. If $\widehat{\nu}_{0}$ satisfies

$$
\widehat{\nu}_{0}\left(\xi_{l}\right) \geq 0, \quad l=0, \ldots, N-1,
$$

then for all $k>0$

$$
\widehat{\nu}_{l}^{k} \geq 0, \quad l=0, \ldots, N-1 .
$$

Proof. Here, we restrict ourselves to the case where the sequence $\left(\xi_{l}\right)_{l}$ belongs to $\left[\xi_{1}, \xi_{2}\right]$. The discretization of $\widehat{\nu}$ is given by

$$
\widehat{\nu}_{l}^{k+1}=\operatorname{sgn}\left(\xi_{l}\right) \frac{H_{l+\frac{1}{2}}^{k+1}-H_{l-\frac{1}{2}}^{k+1}}{\triangle \xi} .
$$


The implicit difference scheme used for $H$ implies that the sequence $\left(\widehat{\nu}_{j}^{k}\right)_{(j, k)}$ satisfies

$$
-\frac{\triangle t}{\triangle \xi^{2}} \xi_{l+\frac{1}{2}}^{2} \beta_{l+1}^{k} \widehat{\nu}_{l+1}^{k+1}+\left(1+\frac{\triangle t}{\triangle \xi^{2}} \beta_{l}^{k}\left(\xi_{l+\frac{1}{2}}^{2}+\xi_{l-\frac{1}{2}}^{2}\right)\right) \widehat{\nu}_{l}^{k+1}-\frac{\triangle t}{\triangle \xi^{2}} \xi_{l-\frac{1}{2}}^{2} \beta_{l-1}^{k} \widehat{\nu}_{l-1}^{k+1}=\widehat{\nu}_{l}^{k} .
$$

Suppose that $\widehat{\nu}_{l}^{k} \geq 0$ for all $l$ and introduce the sequence $\mu_{l}^{k+1}$ such that $\mu_{l}^{k+1}=\beta_{l}^{k} \widehat{\nu}_{l}^{k+1}$ and $l_{0}$ such that $\mu_{l_{0}}^{k+1}=\min _{l}\left(\mu_{l}^{k+1}\right)$.

Firstly, suppose that $\mu_{l_{0}}^{k+1}<0$ then $\beta_{l_{0}}^{k} \neq 0$. It follows that

$$
\left(\frac{1}{\beta_{l_{0}}^{k}}+\frac{\triangle t}{\triangle \xi^{2}}\left(\xi_{l_{0}+\frac{1}{2}}^{2}+\xi_{l_{0}-\frac{1}{2}}^{2}\right)\right) \mu_{l_{0}}^{k+1}=\widehat{\nu}_{l_{0}}^{k}+\frac{\triangle t}{\triangle \xi^{2}} \xi_{l_{0}+\frac{1}{2}}^{2} \mu_{l_{0}+1}^{k+1}+\frac{\triangle t}{\triangle \xi^{2}} \xi_{l_{0}-\frac{1}{2}}^{2} \mu_{l_{0}-1}^{k+1} .
$$

Since $\mu_{l_{0}+1}^{k+1} \geq \mu_{l_{0}}^{k+1}$ and $\mu_{l_{0}-1}^{k+1} \geq \mu_{l_{0}}^{k+1},(4.18)$ implies that

$$
\frac{\mu_{l_{0}}^{k+1}}{\beta_{l_{0}}^{k}} \geq \widehat{\nu}_{l_{0}}^{k} \geq 0
$$

that is $\mu_{l_{0}}^{k+1}$ is positive which is a contradiction. So the sequence $\left(\mu_{l}^{k}\right)_{l}$ is positive for all $l$. Therefore the sequence $\left(\widehat{\nu}_{l}^{k+1}\right)_{l}$ is positive for $l$ not included in the set $J=\left\{j\right.$ s.t. $\left.\beta_{j}^{k}=0\right\}$.

Let be $j$ such that $\beta_{j}^{k}=0$, then for all $k \geq 0$

$$
\widehat{\nu}_{j}^{k+1}=\widehat{\nu}_{j}^{k}+\frac{\triangle t}{\triangle \xi^{2}} \xi_{j+\frac{1}{2}}^{2} \mu_{j+1}^{k+1}+\frac{\triangle t}{\triangle \xi^{2}} \xi_{j-\frac{1}{2}}^{2} \mu_{j-1}^{k+1}
$$

which shows that $\widehat{\nu}_{j}^{k+1}$ is positive and the result follows.

\subsection{Time-splitting spectral discretizations for the Zakharov system}

Many numerical methods have been proposed for the Zakharov part of the system. For example Payne et al. [13] have introduced a spectral method. They used a truncated Fourier expansion in their algorithm to eliminate aliasing errors. Glassey [9] presented an energy-preserving finite difference scheme for the Zakharov system in one dimension. Here we describe a time-splitting with a spectral discretization. The first equation (4.1) is solved in two splitting steps. One first solves

$$
i\left(\partial_{t} E+\nu * E\right)+\partial_{x}^{2} E=E_{p}(x) \mathrm{e}^{i\left(k_{1} x-\omega_{1} t\right)},
$$

for the time step of length $\triangle t$. In the second step, one solves

$$
\begin{aligned}
& i \partial_{t} E=\frac{1}{2} \delta n E \\
& \partial_{t}^{2} \delta n-\mu \partial_{x}^{2} \delta n=\mu \partial_{x}^{2}\left(|E|^{2}\right), \\
& E_{0}=E^{*}(.), \\
& \delta n_{0}=\delta n\left(t^{k}, .\right), \\
& \partial_{t} \delta n_{0}=\partial_{t} \delta n\left(t^{k}, .\right) .
\end{aligned}
$$

Equation (4.20) will be discretized in space by the Fourier spectral method and integrated exactly in time. Let $E^{*}$ be the solution of (4.20) at time $t=t_{k+1}$. For $t \in\left[t_{k}, t_{k+1}\right]$, multiplying (4.21) by $\bar{E}$, the conjugate of $E$ 
and taking the imaginary part, we get:

$$
\frac{\mathrm{d}}{\mathrm{d} t}|E(t, x)|^{2}=0
$$

With this conservation law, in order to solve (4.21)-(4.25), we have to solve:

$$
\begin{aligned}
& i \partial_{t} E=\frac{1}{2} n E \\
& \partial_{t}^{2} P-\mu \partial_{x}^{2} P=0 \\
& E_{0}=E^{*}(.) \\
& P_{0}=P\left(t^{k}, .\right) \\
& \partial_{t} P_{0}=\partial_{t} n\left(t^{k}, .\right)=P_{1},
\end{aligned}
$$

with $P=\delta n+\left|E^{*}\right|^{2}$.

Finally, from $t=t_{k}$ to $t=t_{k+1}$, using trapezoidal rule, we combine the splitting steps via the standard Strang splitting.

For the first step of the splitting, we compute

$$
\begin{aligned}
& \widehat{E}_{l}^{k}=\frac{1}{N} \sum_{j=1}^{N} E_{j}^{k} \mathrm{e}^{-i \xi_{l} x_{j}}, l=-M, \ldots, M-1, \\
& \widehat{E}_{l}^{*}=\widehat{E}_{l}^{k} \exp \left(-\left(\widehat{\nu}_{l}^{k}+\widehat{\nu}_{l}^{k+1}\right) \frac{\triangle t}{2}+\frac{3 i \xi_{l}^{2}}{2} \triangle t\right) \\
& \left.+\frac{\delta t}{2}\left(\widehat{f}_{l}^{k} \exp \left(-\left(\widehat{\nu}_{l}^{k}+\widehat{\nu}_{l}^{k+1}\right) \frac{\triangle t}{2}+\frac{3 i \xi_{l}^{2}}{2} \triangle t\right)+\widehat{f}_{l}^{k+1}\right)\right), l=-M, \ldots, M-1, \\
& E_{j}^{*}=\sum_{l=-M}^{M-1} \widehat{E}_{l}^{*} \mathrm{e}^{i \xi_{l} x_{j}}, \quad j=1, \ldots, N,
\end{aligned}
$$

where $f=E_{p}(x) \mathrm{e}^{i\left(k_{1} x-\omega_{1} t\right)}$ and $(\widehat{U})_{l}$ denote the Fourier coefficients of the periodic vector $U=\left(U_{0}, U_{1}, \ldots, U_{N}\right)^{T}$.

For the second step, we compute

$$
\begin{aligned}
& \widehat{P}_{l}^{k+1}=\widehat{P}_{0}\left(\xi_{l}\right) \cos \left(\sqrt{\mu} \xi_{l} \triangle t\right)+\widehat{P}_{1}\left(\xi_{l}\right) \frac{\sin \left(\sqrt{\mu} \xi_{l} \triangle t\right)}{\sqrt{\mu} \xi_{l}}, \\
& \partial_{t} \widehat{P}_{l}^{k+1}=\widehat{P}_{1}\left(\xi_{l}\right) \cos \left(\sqrt{\mu} \xi_{l} \triangle t\right)-\sqrt{\mu} \xi_{l} \widehat{P}_{0}\left(\xi_{l}\right) \sin \left(\sqrt{\mu} \xi_{l} \triangle t\right), \\
& P_{j}^{k+1}=\sum_{l=-M}^{M-1} \widehat{P}_{l}^{k+1} \mathrm{e}^{i \xi_{l} x_{j}}, \quad j=1, \ldots, N, \\
& \delta n_{j}\left(t^{k+1}\right)=P_{j}^{k+1}-\left|E_{j}^{*}\right|^{2}, \quad j=1, \ldots, N, \\
& E_{j}^{k+1}=E_{j}^{*} \exp \left(-\frac{i}{2} \int_{t^{k}}^{t^{k+1}} \delta n_{j}(s) \mathrm{d} s\right) \simeq E_{j}^{*} \exp \left(-\frac{i}{2}\left(\delta n_{j}^{k}+\delta n_{j}^{k+1}\right) \frac{\triangle t}{2}\right) .
\end{aligned}
$$


Now let $\|\cdot\|_{l^{2}}$ be the usual discrete $l^{2}$ norm on the interval $(0, L)$ :

$$
\|U\|_{l^{2}}=\sqrt{\frac{L}{N} \sum_{j=1}^{L}\left|U_{j}\right|^{2}}
$$

then, we have

Proposition 4.4. ( $l^{2}$ stability). If the initial data $\widehat{\nu}_{0}$ and $n_{1}()=.\partial_{t} \delta n(., 0)$ satisfy

$$
\widehat{\nu}_{0}\left(\xi_{j}\right) \geq 0 j=0, \ldots, N-1, \quad \text { and } \int n_{1}(x) \mathrm{d} x=0
$$

then the time-splitting spectral discretization of the Zakharov system have the following properties:

$$
\begin{gathered}
\left\|E^{k+1}\right\|_{l^{2}}^{2} \leq\left\|E^{k}\right\|_{l^{2}}^{2}+\Delta t^{2}\left\|E_{p}\right\|_{l_{2}}^{2} \quad \forall k>0, \\
\frac{b-a}{N} \sum_{j=1}^{N} \delta n_{j}^{k}=\frac{b-a}{N} \sum_{j=1}^{N} \delta n_{j}^{0}=\frac{b-a}{N} \sum_{j=1}^{N} \delta n_{0}\left(x_{j}\right), k=1,2, \ldots
\end{gathered}
$$

Proof.

1. $l_{2}$ estimate (4.36):

We multiply equation (4.35) by $\bar{E}^{k+1}$ and obtain:

$$
\begin{aligned}
\frac{N}{L}\left\|E^{k+1}\right\|_{l_{2}}^{2}= & \sum_{j=1}^{N}\left|E_{j}^{k+1}\right|^{2}=\sum_{j=1}^{N}\left|E_{j}^{*} \exp \left(-i\left(\delta n_{j}^{k}+\delta n_{j}^{k+1}\right) \frac{\delta t}{2}\right)\right|^{2}, \\
= & \sum_{j=1}^{N}\left|E_{j}^{*}\right|^{2}=\sum_{j=1}^{N}\left|\sum_{l=-M}^{M-1} \widehat{E}_{l}^{*} \mathrm{e}^{i \xi_{l} x_{j}}\right|^{2}, \\
= & \sum_{j=1}^{N}\left(\sum_{-M \leq l, k \leq M-1}\left(\widehat{E}^{*}\right)_{l} \overline{\left(\widehat{E}^{*}\right)_{k}} \mathrm{e}^{i\left(\xi_{l}-\xi_{k}\right) x_{j}}\right) \\
= & \sum_{-M \leq l, k \leq M-1}\left(\sum_{j=1}^{N} \mathrm{e}^{i\left(\xi_{l}-\xi_{k}\right) x_{j}}\right)\left(\widehat{E}^{*}\right)_{l} \overline{\left(\widehat{E}^{*}\right)_{k}}
\end{aligned}
$$

Then with $\sum_{j=1}^{N} \mathrm{e}^{i\left(\xi_{l}-\xi_{k}\right) x_{j}}=\sum_{j=1}^{N} \mathrm{e}^{\frac{2 i \pi}{N}(l-k)(j-1)}=\left\{\begin{array}{l}N \text { if } k-l=p N \\ 0 \text { if } k-l \neq p N\end{array}\right.$, we have

$$
\frac{N}{L}\left\|E^{k+1}\right\|_{l_{2}}^{2}=N \sum_{l=-M}^{M-1}\left|\left(\widehat{E}^{*}\right)_{l}\right|^{2}=N \sum_{l=-M}^{M-1}\left|\exp \left(\frac{3}{2} i \xi_{l}^{2} \delta t-\left(\hat{\nu}_{l}^{k}+\hat{\nu}_{l}^{k+1}\right) \frac{\delta t}{2}\right)\left(\widehat{E}^{k}\right)_{l}\right|^{2}
$$

Using Proposition 4.3, we obtain that

$$
\frac{N}{L}\left\|E^{k+1}\right\|_{l_{2}}^{2} \leq N \sum_{l=-M}^{M-1}\left|\left(\widehat{E}^{k}\right)_{l}\right|^{2}
$$


so

$$
\frac{N}{L}\left\|E^{k+1}\right\|_{l_{2}}^{2} \leq \sum_{j=1}^{N}\left|E_{j}^{k}\right|^{2}=\frac{N}{L}\left\|E^{k}\right\|_{l_{2}}^{2} .
$$

2. Low-frequency density conservation.

Since we have the relation

$$
\widehat{\delta} n\left(\xi_{0}\right)=\frac{1}{N} \sum_{j=1}^{N} \delta n_{j},
$$

with $\xi_{0}=0$, it is enough to show that $\widehat{\delta n}\left(\xi_{0}\right)$ is preserved.

For that, one uses the expressions given previously for $\widehat{P}_{j}^{k+1}$ and $\partial_{t} \widehat{P}_{j}^{k+1}$ with $P_{j}=\delta n_{j}+\frac{1}{4}\left|E_{j}^{*}\right|^{2}$.

Taking (4.31) for $\xi=\xi_{0}$ yields

$$
\partial_{t} \widehat{P}^{k+1}\left(\xi_{0}\right)=\partial_{t} \widehat{P}^{k}\left(\xi_{0}\right)
$$

Then, by using the hypothesis on the initial data $\delta n_{1}($.$) , we have$

$$
\partial_{t} \widehat{P}^{k}\left(\xi_{0}\right)=0 \forall k>0
$$

Taking (4.32) for $\xi=\xi_{0}$ yields

$$
\widehat{P}^{k+1}\left(\xi_{0}\right)=\widehat{P_{0}}\left(\xi_{0}\right)=\widehat{P}^{k}\left(\xi_{0}\right)
$$

So

$$
\widehat{\delta n}^{k+1}\left(\xi_{0}\right)+\frac{1}{4} \mathcal{F}_{d}\left(\left|E^{*}\right|^{2}\right)\left(\xi_{0}\right)=\widehat{\delta n}^{k}\left(\xi_{0}\right)+\frac{1}{4} \mathcal{F}_{d}\left(\left|E^{*}\right|^{2}\right)\left(\xi_{0}\right),
$$

where $\mathcal{F}_{d}$ denotes the discret Fourier transform.

Therefore we have

$$
\sum_{j=1}^{N} \delta n_{j}^{k+1}=\sum_{j=1}^{N} \delta n_{j}^{k}
$$

and the result follows.

\subsection{Numerical results}

\subsubsection{The quasi-linear diffusion}

In order to illustrate how the quasi-linear diffusion works, we computed the diffusion equation with a diffusion coefficient given by a fixed electric field

$$
E(x)=\mathrm{e}^{i k_{1} x} \mathrm{e}^{-\frac{\left(x-\frac{L}{2}\right)^{2}}{2 \beta^{2}}}
$$

with $L=2000, \beta=50$ and $k_{1}=0.45$.

The initial electron distribution function is assumed to be a Maxwellian,

$$
F_{e 0}(v)=\frac{1}{\sqrt{2 \pi}} \exp \left(-\frac{v^{2}}{2}\right) .
$$

We can remark that the electron function is flattened near the phase velocity $v_{\phi}=\frac{1}{k_{1}}=2.22$ and since the Landau damping rate depends on the slope of the electron distribution, we can see that $\widehat{\nu}$ tends toward zero near $\xi=k_{1}$. 

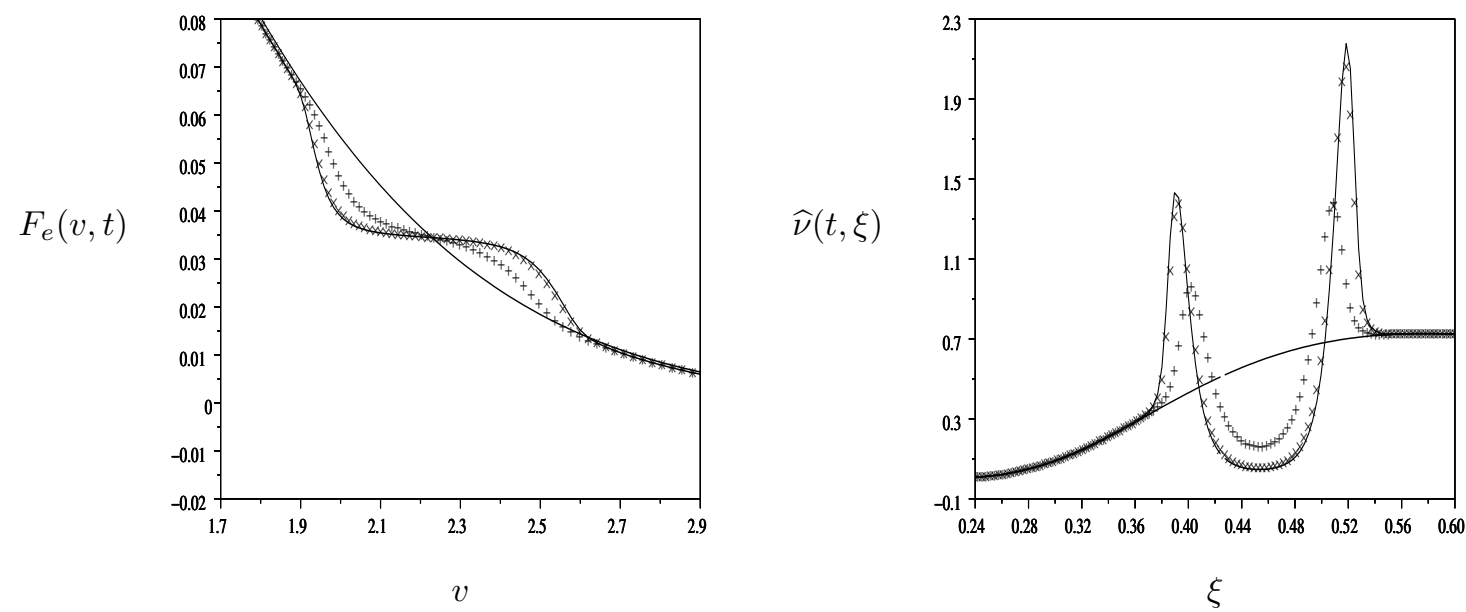

Figure 1. The left plot corresponds to the evolution of the electron function distribution and the right plot to the evolution of the Landau damping rate.

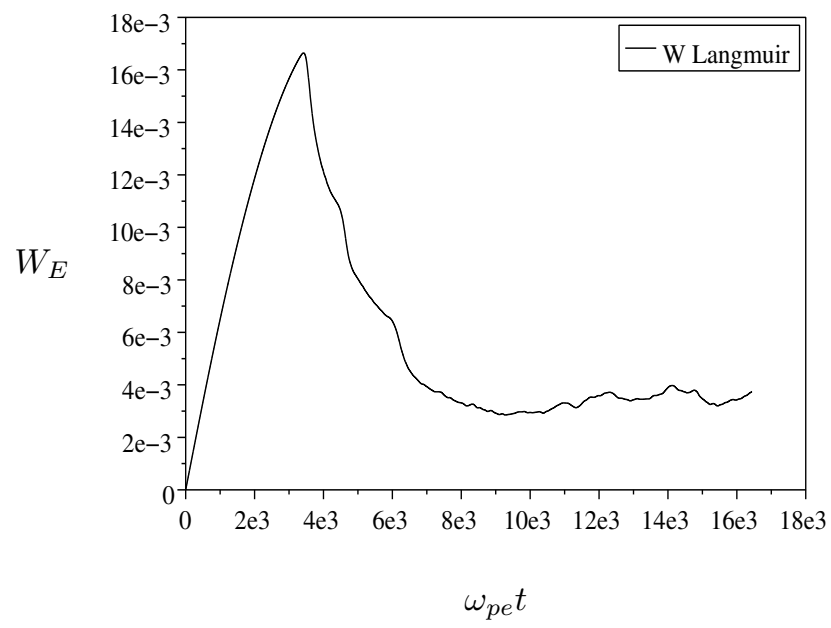

Figure 2. The time history of the spatially averaged Langmuir wave energy $|E|^{2}$ with $\left|E_{p}\right|=$ $8.10^{-3}$ and $k_{1} \lambda_{D e}=0.09$.

\subsubsection{The full system}

In this part, simulation results using the above quasi-linear Zakharov (4.1)-(4.4) model is presented. The mass ratio is $\sqrt{m_{e} / m_{i}}=1 / 50$ and the simulation box length is $L=2000 \lambda_{D e}$ (where $\lambda_{D e}$ denotes the Debye length).

The initial electric field and low frequency density in the quasi-linear Zakharov simulation are equal to zero. The initial electron distribution function is assumed to be a Maxwellian.

The system is driven with an external periodic force localized in the center of the box and we use a 1024 grid point with $\triangle \xi=2 \pi / L$.

We present the results for $k_{1} \lambda_{D e}=0.09$ and $\left|E_{p}\right|=8.10^{-3}$ which corresponds to a weakly driven case.

Figure 2 shows the time history of the spatially averaged Langmuir wave energy: $W_{E}=\int|E|^{2} \mathrm{~d} x$. We can see two stages in this simulation: a linear stage where the external pump excited primary electron plasma wave 


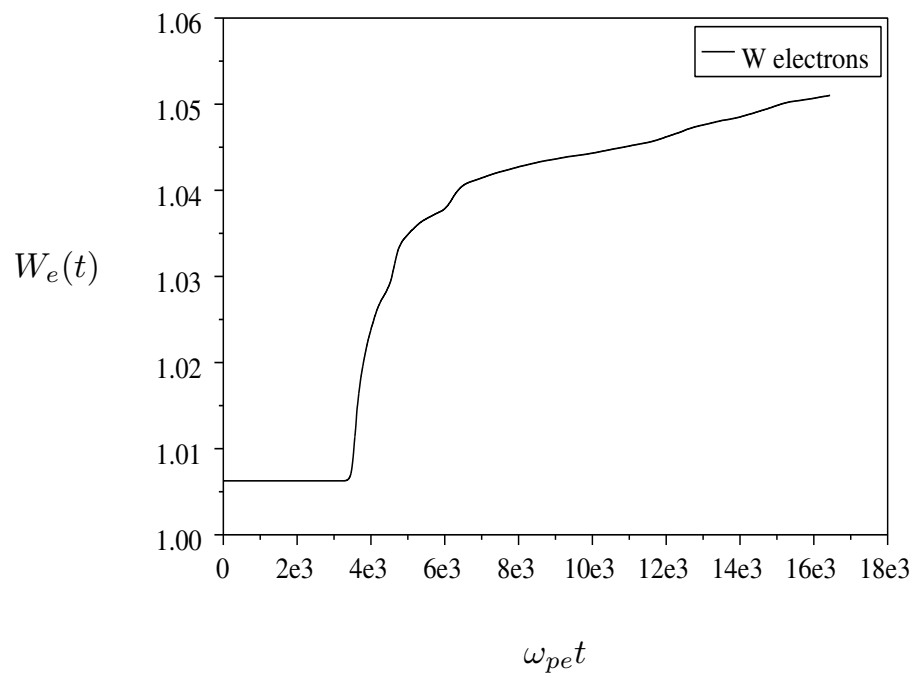

Figure 3 . The time history $W_{e}(t)$ of electron energy with $\left|E_{p}\right|=8.10^{-3}$ and $k_{1} \lambda_{D e}=0.09$.

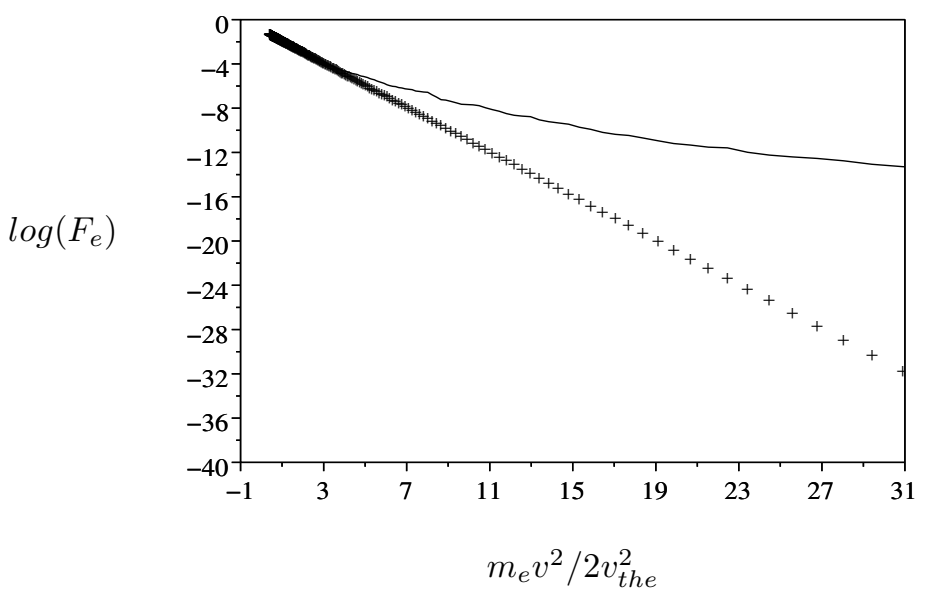

FiguRE 4 . The solid line corresponds to the Log of the spatially averaged electron distribution versus the electron energy $m_{e} v^{2} / 2 v_{\text {the }}^{2}$ at the final time $\omega_{p e} t=16000$ with $|S| / \sqrt{4 \pi n_{0} T_{e}}=$ $8.10^{-3}$ and $k_{p} \lambda_{D e}=0.09$ and the dashed line corresponds to the initial electron distribution.

at $k_{p}$ and a nonlinear stage from $\omega_{p e} t=3000$ where the Langmuir waves have lost energy. Then the system saturates near $\omega_{p e} t=6000$, due to a combination of wave-wave and wave-particle processes. The time history of electron's energy $W_{e}(t)=\int v^{2} F_{e}(v, t) \mathrm{d} v$ versus time in Figure 3 shows us that wave-wave saturation and wave-particle saturation occur simultaneously. Moreover we can estimate that the fraction of pump's energy which heats electrons is $5 \%$. This heating implies that hot electrons have been created. Figure 4 shows the spatially averaged electron distribution at the final time of the simulation. The log of the distribution is plotted with respect to the half square of the velocity. We see significant heating of the electrons: a hot electron tail is produced with approximately 8 times the initial temperature. Concerning the wave-wave process, the Langmuir wave envelope and ion acoustic spectra are shown in Figure 5. Finally, in Figure 6 the electric field amplitude $|E|$ and density perturbation $\delta n$ are spread out spatially and contains many localized intense regions. 

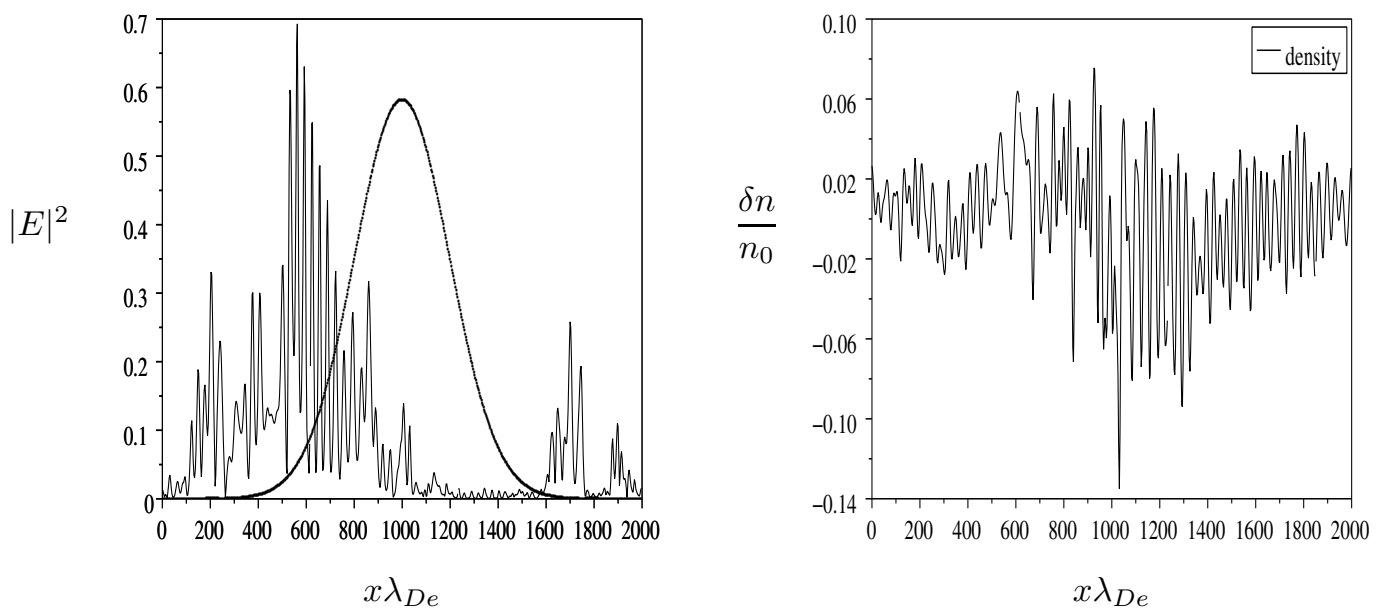

Figure 5. The left plot corresponds to the Langmuir wave in real space and the right plot corresponds to the low frequency density in real space.
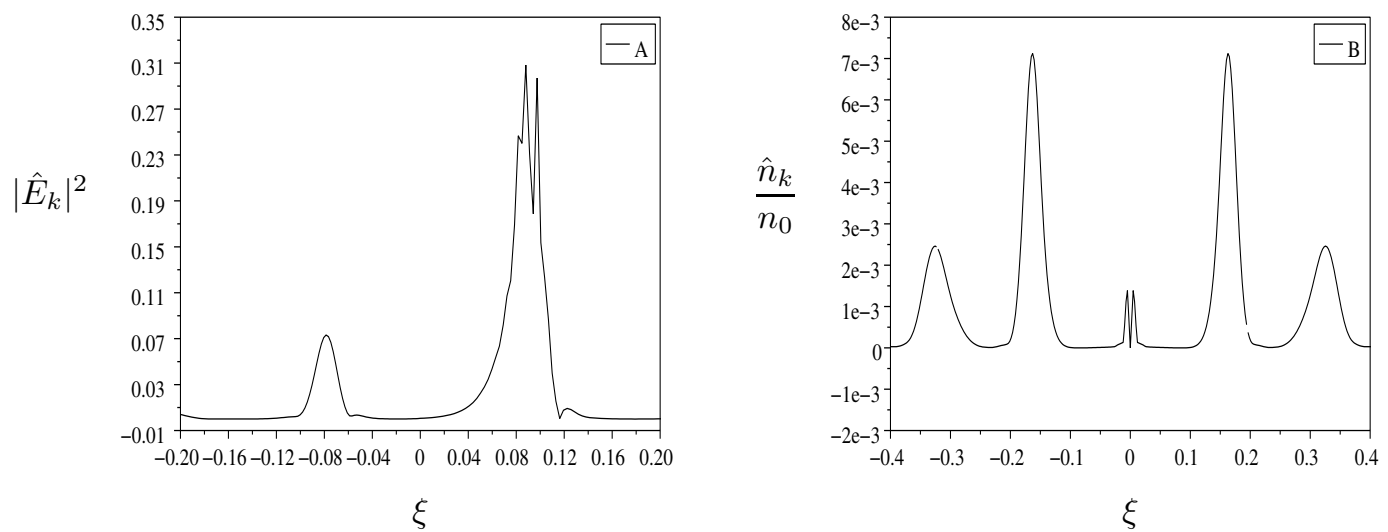

Figure 6. $(A)$ corresponds to the Langmuir envelope spectra and $(B)$ corresponds to the ion-acoustic spectra at the beginning of nonlinear processes.
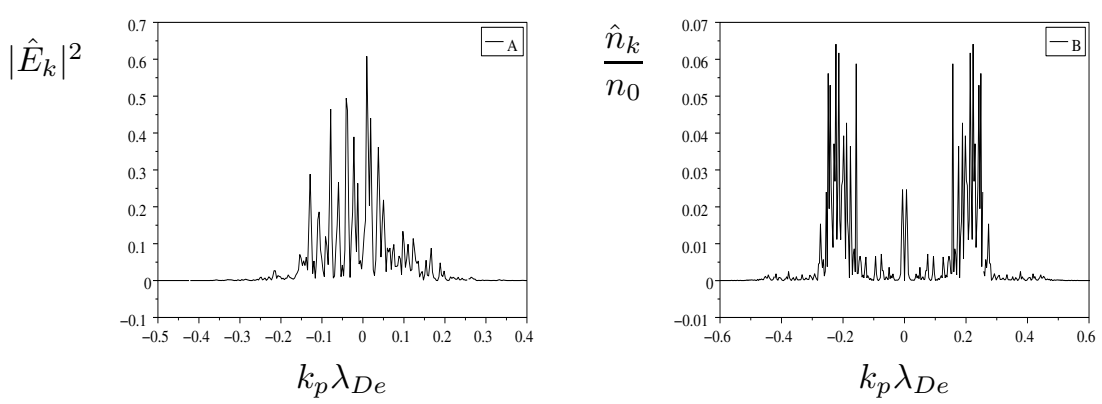

FiguRE 7. $(A)$ corresponds to the Langmuir envelope spectra and $(B)$ corresponds to the ion-acoustic spectra at the final time $\omega_{p e} t=16000$. 
Acknowledgements. The authors are grateful to V. Tikhonchuk for helpful discussions and suggestions concerning the results of this paper.

\section{REFERENCES}

[1] H. Added and S. Added, Equation of Langmuir turbulence and nonlinear Schrödinger equation: smoothness and approximation. J. Funct. Anal. 79 (1988) 183-210.

[2] B. Bidégaray, On a nonlocal Zakharov equation. Nonlinear Anal. 25 (1995) 247-278.

[3] M. Colin and T. Colin, On a quasilinear Zakharov System describing laser-plasma interactions. Diff. Int. Eqs. 17 (2004) $297-330$.

[4] T. Colin and G. Metivier, Instabilities in Zakharov Equations for Laser Propagation in a Plasma, Phase Space Analysis of PDEs, A. Bove, F. Colombini, and D. Del Santo, Eds., Progress in Nonlinear Differential Equations and Their Applications, Birkhauser (2006).

[5] J.-L. Delcroix and A. Bers, Physique des plasmas 1, 2. Inter Editions-Editions du CNRS (1994).

[6] J. Ginibre, Y. Tsutsumi and G. Velo, On the Cauchy problem for the Zakharov system. J. Funct. Anal. 151 (1997) $384-436$.

[7] L. Glangetas and F. Merle, Existence of self-similar blow-up solutions for Zakharov equation in dimension two. I. Comm. Math. Phys. 160 (1994) 173-215.

[8] L. Glangetas and F. Merle, Concentration properties of blow up solutions and instability results for Zakharov equation in dimension two. II. Comm. Math. Phys. 160 (1994) 349-389.

[9] R.T. Glassey, Convergence of an energy-preserving scheme for the Zakharov equations in one space dimension. Math. Comp. 58 (1992) 83-102.

[10] C.E. Kenig, G. Ponce and L. Vega, Smoothing effects and local existence theory for the generalized nonlinear Schrödinger equations. Invent. Math. 134 (1998) 489-545.

[11] F. Linares, G. Ponce and J.C. Saut, On a degenerate Zakharov system. Bull. Braz. Math. Soc. New Series 36 (2005) 1-23.

[12] T. Ozawa and Y. Tsutsumi, Existence and smoothing effect of solution for the Zakharov equations. Publ. Res. Inst. Math. Sci. 28 (1992) 329-361.

[13] G.L. Payne, D.R. Nicholson and R.M. Downie, Numerical Solution of the Zakharov Equations. J. Compt. Phys. 50 (1983) $482-498$.

[14] G. Riazuelo. Étude théorique et numérique de l'influence du lissage optique sur la filamentation des faisceaux lasers dans les plasmas sous-critiques de fusion inertielle. Ph.D. thesis, University of Paris XI.

[15] D.A. Russel, D.F. Dubois and H.A. Rose. Nonlinear saturation of simulated Raman scattering in laser hot spots. Phys. Plasmas 6 (1999) 1294-1317.

[16] K.Y. Sanbomatsu, Competition between Langmuir wave-wave and wave-particule interactions. Ph.D. thesis, University of Colorado, Department of Astrophysical (1997).

[17] S. Schochet and M. Weinstein, The nonlinear Schrödinger limit of the Zakharov equations governing Langmuir turbulence. Comm. Math. Phys. 106 (1986) 569-580.

[18] C. Sulem and P.-L. Sulem, Quelques résultats de régularité pour les équations de la turbulence de Langmuir. C. R. Acad. Sci. Paris Sér. A-B 289 (1979) 173-176.

[19] C. Sulem and P.-L. Sulem, The nonlinear Schrödinger Equation. Self-Focusing and Wave Collapse. Appl. Math. Sci. 139, Springer (1999).

[20] B. Texier, Derivation of the Zakharov equations. Arch. Rat. Mech. Anal. (to appear).

$[21]$ V.E. Zakharov, S.L. Musher and A.M. Rubenchik, Hamiltonian approach to the description of nonlinear plasma phenomena. Phys. Reports 129 (1985) 285-366. 